\section{Information Systems Research}

Articles in Advance, pp. 1-21

ISSN 1047-7047 (print) | ISSN 1526-5536 (online) http:/ / dx.doi.org/10.1287/isre.1110.0415 (C) 2012 INFORMS

\title{
Real-Time Tactical and Strategic Sales Management for Intelligent Agents Guided by Economic Regimes
}

\author{
Wolfgang Ketter \\ Rotterdam School of Management, Erasmus University, 3062 PA Rotterdam, The Netherlands, \\ wketter@rsm.nl \\ John Collins, Maria Gini \\ Computer Science and Engineering, University of Minnesota, Minneapolis, MN 55455, \\ \{jcollins@cs.umn.edu, gini@cs.umn.edu\} \\ Alok Gupta \\ Carlson School of Management, University of Minnesota, Minneapolis, MN 55455, alok@umn.edu \\ Paul Schrater \\ Computer Science and Engineering, University of Minnesota, Minneapolis, MN 55455, schrater@umn.edu
}

\begin{abstract}
$\mathrm{M}^{2}$ any enterprises that participate in dynamic markets need to make product pricing and inventory resource utilization decisions in real time. We describe a family of statistical models that addresses these needs by combining characterization of the economic environment with the ability to predict future economic conditions to make tactical (short-term) decisions, such as product pricing, and strategic (long-term) decisions, such as level of finished goods inventories. Our models characterize economic conditions, called economic regimes, in the form of recurrent statistical patterns that have clear qualitative interpretations. We show how these models can be used to predict prices, price trends, and the probability of receiving a customer order at a given price. These "regime" models are developed using statistical analysis of historical data and are used in real time to characterize observed market conditions and predict the evolution of market conditions over multiple time scales. We evaluate our models using a testbed derived from the Trading Agent Competition for Supply Chain Management, a supply chain environment characterized by competitive procurement, sales markets, and dynamic pricing. We show how regime models can be used to inform both short-term pricing decisions and long-term resource allocation decisions. Results show that our method outperforms more traditional short- and long-term predictive modeling approaches.

Key words: enabling technologies; agent-mediated electronic commerce; dynamic pricing; price forecasting; economic regimes; supply chain; dynamic markets; trading agent competition

History: Chris Dellarocas, Senior Editor; Danial Zeng, Associate Editor. This paper was received on August 14, 2009, and was with the authors 14 months for 3 revisions. Published online in Articles in Advance.
\end{abstract}

\section{Introduction}

To seek competitive advantage, firms are employing increasingly sophisticated automated decision support systems. These advanced decision support systems often involve designing software agents that can act rationally on behalf of their users or assist the users in a variety of application areas. Examples include procurement (Sandholm 2007), scheduling and resource management (Collins et al. 2002), and personal information management (Berry et al. 2006, Mark and Perrault 2006). Software agents have the advantage of being able to analyze many more possibilities in shorter time frames than their human counterparts but are often limited in their ability to make strategic decisions. In this paper we present computational methods for a software agent that considers long-term expected profit implications when making short-term tactical decisions, such as setting current prices and quantities of products to sell in a given time frame.

We look at a complex and critical part of the supply chain relating to product pricing decisions in an auction based dynamic pricing environment where customer demand is stochastic. We are particularly interested in multicommodity supply chain environments that are constrained by capacity and materials availability and where market conditions may be characterized qualitatively, for example, by oversupply or scarcity. Such environments exist in businessto-business (B2B) exchanges where several suppliers compete for business from customers for commoditized manufactured parts (Kaplan and Sawhney 2000). Given the rapid increase in implementations of 
technology assisted market based mechanisms, in the near future such environments are likely to develop for more complex products.

One of the innovative and unique characteristics of our approach is to make pricing decisions based not only on current demand but also on anticipated future demand and other hidden factors, which are aggregated by assessing the "economic regimes" and their expected future transitions. Economic regimes characterize market conditions by detecting distinguishable statistical patterns in historical market data. They capture overall market conditions, such as scarcity or oversupply, and provide valuable indications such as price trend and price distribution predictions over a planning horizon. In this work, we focus on observable pricing data as a surrogate for a range of typically hidden variables that affect pricing decisions of buyers and sellers in a market.

In previous research (Ketter et al. 2009) we proposed the use of economic regimes and showed how to identify them from historical data. However, we did not address whether regime predictions can be made for new unseen environments. Further it was not clear how managerial decisions such as pricing, sales quota, and profits could benefit from the knowledge of economic regime forecasts. These issues are addressed in this paper, where we present new methods to identify economic regimes in real time and to predict future regime transitions and related future price distributions and price trends. Our computational approaches are lightweight, i.e., designed to operate with minimal computational burden so that they can respond to requests in real-time.

Further, we develop a model that uses regime predictions to set sales quotas for current and future sales with the objective of maximizing profit over time. Our approach is tested by embedding our computational methods in a software agent that operates in the Trading Agent Competition for Supply Chain Management (TAC SCM; Collins et al. 2010b). Experimental results show that our approach performs better than traditional predictive modeling methods.

Although predictions about the economic environment are commonly made at the macroeconomic level (Osborn and Sensier 2002), to our knowledge, such predictions are rarely done for microeconomic environments and represent a novel contribution of this research. In addition, systemic use of these forecasts for decision making is also a unique contribution of this research. Our previous work (Ketter et al. 2009) focused on using economic regimes for their explanatory power, whereas this paper focuses on their predictive power. This distinction is central to the current debate on explanatory versus predictive modeling (Shmueli 2010).
Economic regimes can be used to support decisions in both procurement and sales markets. In the procurement market, we may have little or no control over the availability of parts, but we can control the usable supply to a certain degree. Prices increase when there is scarcity. Scarcity of parts commonly results from excess demand, which tends to occur when demand for associated products is high. This is precisely why prediction of regimes is important. If we can predict an increase in prices of finished products, then we may decide to acquire parts early, thereby reducing cost of material and increasing our profit margin.

The approach we present is applicable also to commodity markets for items such as cotton, oil, or semiconductor chips, where fast changing market conditions and high price volatility are common. For example, the procurement risk management process at Hewlett-Packard (Nagali et al. 2008) uses probabilistic estimates of future price, demand, and supply to forecast a range of future market scenarios, which are in turn used to evaluate potential procurement contracts. This process has saved Hewlett-Packard hundreds of millions of dollars in the procurement of flash memory alone. Although Nagali et al. (2008) do not describe their price prediction model in detail, the regimebased model we describe in this paper produces a probabilistic estimate of future prices that could be used in such a system. Our results show that even though a market may be constantly changing, there are some underlying dominant patterns or economic regimes that characterize market conditions.

The paper is organized as follows. In §2 we review relevant literature. Section 3 describes the foundations of our economic regime approach. It shows how to make real-time predictions about future economic regimes and price distributions and how economic regimes can support strategic and tactical sales decisions. Section 4 describes our testbed, the TAC SCM. In $\S 5$ we present experimental results using the TAC SCM testbed. Finally, we conclude with directions for future research.

\section{Background and Literature Review}

Pricing of products to retailers or distributors is a key aspect of supply chain management for any profit maximizing firm. Most studies (e.g., Cachon and Netessine 2004, Kleindorfer and $\mathrm{Wu} 2003$ ) look at this issue in a single supplier and single buyer setting because of analytical complexity and tractability issues. Although dynamic pricing is seen as a potentially superior approach (Elmaghraby and Keskinocak 2003, Swaminathan and Tayur 2003), the market power, and thus the power to set prices, is still assumed to be with the supplier or manufacturer. However, information systems researchers have started 
looking at the potential of dynamic pricing through auction based approaches to provide incentives for supply chain coordination (e.g., Fan et al. 2003). Our approach is based on the assumption that competitive markets where manufacturers compete for customers' business will eventually lead to dynamic pricing, in which prices will emerge from interactions between manufacturers and their customers.

Various methods to predict prices have been used, such as in first price sealed bid reverse auctions for IBM PCs (Lawrence 2003) and PDAs on eBay (Ghani 2005) or in predicting ending prices for a multi-unit online ascending auction (Bapna et al. 2008). Dynamic forecasting of auction bidding prices is becoming increasingly popular because of the massive use of online auctions (Wang et al. 2008). Short-term price prediction has been the focus of several studies where prices move primarily because of demand-side constraints, such as in the electricity market (Nogales et al. 2002). Specific methods for price prediction in TAC SCM are covered later in $\$ 4.1$.

Although approaches to price prediction vary considerably, it is widely recognized that predictions need to exploit the information available in the market and to take its structure into account (Muth 1961). However, as Gray and Spencer (1990) note, demand side price movements are intrinsically linked with supply side movements. Massey and Wu (2005) show that the ability of decision makers to correctly identify the onset of a new regime can mean the difference between success and failure. Furthermore, they found strong evidence that individuals pay inordinate attention to the signal (price in our case) and neglect the aspects of the system that generate the signal (regime dynamics). This results in a tendency to over- or underreact to market conditions.

Several researchers have identified the existence and cyclic nature of economic regimes in consumer markets. For example, Ghose et al. (2006) empirically analyze the degree to which used products cannibalize new product sales for books on Amazon.com and show that product prices go through different regimes over time. Similarly, Pauwels and Hanssens (2002) analyze how strategic windows of change alternate with long periods of stability in mature economic markets.

In this paper we develop computationally efficient methods to identify and predict economic regimes that can be used by decision makers or by autonomous computational agents to make pricing decisions in a complex supply chain environment. Our method is able to detect and forecast a broad range of market conditions. Regression based approaches (including nonparametric variations) assume that the functional form of the relationship between dependent and independent variables has a consistent structure across the range of market conditions. In contrast, our approach models variability in market conditions and does not assume a functional relationship; this allows detection of changes in relationship between prices and sales over time.

\section{Economic Regimes for Real-Time Prediction of Price Distributions}

We now describe the details of our approach. Any economic decision process should account for prevailing and future market conditions because these changing conditions affect an organization's strategies for procurement, production planning, and pricing. These market conditions can be broadly defined as scarcity, balanced, and oversupply. A scarcity condition exists when demand exceeds product supply in the market, a balanced condition when demand is approximately equal to supply, and an oversupply condition when supply exceeds demand. When there is scarcity, firms have pricing power and may price more aggressively. In balanced situations, prices have some spread, so firms have a range of options for maximizing expected profit. In oversupply situations, prices are lower and firms should primarily control costs and therefore either price based on costs or conserve resources for better market conditions.

As indicated earlier, we assume that observable prices act as signals of the underlying true state of the economy, and we use them to estimate future regimes, from which we can then estimate price trends and price distributions. Our regime model is a Hierarchical Hidden Markov Model (HHMM; Fine et al. 1998). A HHMM allows for the existence of hidden, as well as observable, parameters. Price is an observable parameter whose changes drive a hidden "state" (economic regime) of the economy.

Overall, the computational approaches we present are able to

1. identify the current economic regime using price history and real-time data;

2. estimate future regimes of the market, specifically regime distributions, price density, price trends, and probability of receiving orders at a given price; and

3. make dynamic decisions on what products to sell and at what price using the predictions.

\subsection{Background}

We focus our work on an exchange marketplace that is characterized by several competing firms offering several identical products; because the products are identical, customers' buying decisions are only based on price. We assume that during each discrete planning period (which we call "day") each firm decides whether or not to offer a product and set an appropriate price for each product that is offered. Such 
decisions require projecting future customer demand along with a given firm's inventory levels, production capacity, and other necessary resources.

For simplicity, we aggregate price data for different goods. Because prices may have different ranges for different products, we normalize them by dividing the price of a good by the nominal cost of its components and the variable assembly cost. We assume prices are dynamic and change every day according to market conditions. We define the normalized price for good $g$ on day $d$ as $\mathrm{np}_{d, g}=$ price $_{d, g} /$ (nominal_cost . $\left(\mathscr{C}_{g}\right)+$ qassembly_cost $\left.t_{g}\right)$. where $\mathscr{C}_{g}$ is the set of components in product $g$. In the following, for simplicity of notation, we use $\mathrm{np}$ instead of $\mathrm{np}_{d, g}$, unless there is ambiguity.

We briefly summarize the theory of economic regimes (Ketter et al. 2009) as a foundation for the rest of this paper. Instead of assuming a given distribution for prices, we approximate an arbitrary price distribution by fitting a Gaussian mixture model (GMM; Titterington et al. 1985) to historical normalized price data. The demand characteristics in electronic marketplaces have been found to be fractal; that is, the short-term demand pattern has much larger variation than does the long-term time-averaged demand pattern (Gupta et al. 1997). This means that although there are periods of no or little demand, there will be periods when demand will be extremely high. The pricing strategy needs to take this into account. Typically, parameterized econometric models perform poorly in these situations. In contrast, nonparametric approaches do an excellent job in estimation but usually are computationally too expensive. In our testbed and in many real-world trading scenarios, decisions have to be made quickly and there is no time for time consuming calculations. Therefore, we decided to adopt a semi-parametric approach, and in particular the GMM, which can be computed efficiently and uses less memory than other approaches do. ${ }^{1}$

We use the Expectation-Maximization (EM) algorithm (Dempster et al. 1977) to determine the prior probability, $P\left(\zeta_{i}\right)$, of each Gaussian component $\zeta_{i}$ of the GMM. The prior probabilities of these Gaussian components determine the amplitude of a particular Gaussian, and the sum over all Gaussians results in a GMM which fits the underlying data. The density of the normalized price can be written as

$$
p(\mathrm{np})=\sum_{i=1}^{N} p\left(\mathrm{np} \mid \zeta_{i}\right) P\left(\zeta_{i}\right)
$$

\footnotetext{
${ }^{1}$ For a more detailed discussion of the choice and advantages of the chosen modeling approach related to real-time adaptation and decision making, please see the online appendix, available from the Information Systems Research website at http://isr.journal.informs .org/ecompanion.html.
}

where $N$ is the number of Gaussians in the mixture model and $p\left(\mathrm{np} \mid \zeta_{i}\right)$ is the contribution of the $i$ th Gaussian to the normalized price density. The number of Gaussians has to be chosen to balance two conflicting requirements: too many Gaussians will overfit the data and result in a model that does not generalize, whereas too few will provide a crude and inaccurate estimate.

Using Bayes' rule we determine the posterior probabilities for each Gaussian $\zeta_{i}$. We then define the posterior probabilities of all Gaussians given the normalized price $\mathrm{np}$ as the $N$-dimensional vector $\vec{\eta}(\mathrm{np})=\left[P\left(\zeta_{1} \mid \mathrm{np}\right), P\left(\zeta_{2} \mid \mathrm{np}\right), \ldots, P\left(\zeta_{N} \mid \mathrm{np}\right)\right]$. For each observed normalized price $\mathrm{np}_{j}$ we compute the vector of the posterior probabilities, $\vec{\eta}\left(\mathrm{np}_{j}\right)$, which is $\vec{\eta}$ evaluated at each observed normalized price $\mathrm{np}_{j}$. Intuitively, the idea of a regime as a recurrent economic condition is captured by discovering price distributions that recur across time periods in the market. We define regimes by clustering price distributions over time periods using the $k$-means algorithm. The clusters found correspond to frequently occurring price distributions with support on contiguous ranges of $n p$. The center of each cluster is a probability vector that corresponds to a regime $R_{k}$, for $k=1, \ldots, M$, where $M$ is the number of regimes. Collecting these vectors into a matrix yields the conditional probability matrix $\mathbf{P}(\zeta \mid R)$. After we marginalize over all Gaussians $\zeta_{i}$ we obtain the density of the normalized price np dependent on regime $R_{k}$ as

$$
p\left(\mathrm{np} \mid R_{k}\right)=\sum_{i=1}^{N} p\left(\mathrm{np} \mid \zeta_{i}\right) P\left(\zeta_{i} \mid R_{k}\right) .
$$

The probability of regime $R_{k}$ dependent on the normalized price np can then be computed using Bayes' rule as

$$
\begin{aligned}
& P\left(R_{k} \mid \mathrm{np}\right)=\frac{p\left(\mathrm{np} \mid R_{k}\right) P\left(R_{k}\right)}{\sum_{i=1}^{M} p\left(\mathrm{np} \mid R_{i}\right) P\left(R_{i}\right)} \\
& \qquad \text { for } k=1, \ldots, M
\end{aligned}
$$

where $M$ is the number of regimes. The prior probabilities, $P\left(R_{k}\right)$, of the regimes are determined by a counting process over historical data.

At any given time, one of the regimes $R_{k}$ will typically have a higher probability than the others. Economically, it is common to think in terms of three dominant regimes (scarcity, balanced, and oversupply); however, estimating a larger number of regimes can generate additional insights into market conditions, such as extreme oversupply and extreme scarcity. We conducted several experiments varying the number of regimes between 3 and 10 and discovered that 3 and 5 regimes provide the best tradeoff in terms of predictive and explanatory power (Shmueli 2010) and computational load. We use a five regime 
model because the extreme cases (extreme oversupply and extreme scarcity) represent qualitatively distinct market conditions and are therefore important distinctions for decision making. Mathematical details for computing both the optimal number of Gaussians and of regimes are presented in Ketter et al. (2006), Ketter (2007), Ketter et al. (2009), and in the online appendix.

Next we present the computational machinery for real-time predictions, before demonstrating its effectiveness in the TAC SCM environment in $\$ 5$.

\subsection{Real-Time Prediction Methods}

In this section, we describe three different regime prediction methods. The first is based on exponential smoothing, the second is a Markov prediction process, and the last is a Markov correction-prediction process. Each of these methods has different strengths and should be used in different circumstances. The exponential smoother is ideal to estimate the current regime distribution because it makes predictions using only information about the recent past, making it more reactive to the current market condition. The Markov prediction process is appropriate for short- and mid-term predictions, whereas the Markov correction-prediction process is suited for long-term predictions.

\subsubsection{Exponential Smoother Price Prediction.} Using an estimate of the mean normalized price $\tilde{n p}_{d, g}$ (or the actual mean of the normalized price $\mathrm{np}_{d, g}$ if available) for each good $g$ on day $d$, we can compute the price trend and use it to predict future prices. For consistency with the TAC SCM case study we present later, we use the term "day" to refer to a discrete planning period of arbitrary size and we use an estimate of the mean price because the actual mean price is not observable in many markets, including TAC SCM.

Because prices tend to be noisy and both mean and trend vary over time, an exponential smoother can be used to generate short-term predictions from recent observations. Specifically, we use Brown linear exponential smoothing (Brown et al. 1961), which uses two different smoothed series centered at different points in time and a forecasting formula based on an extrapolation of a line through the two centers. The smoothed normalized mean price is computed using $\widetilde{n p^{\prime}}$ and $\widetilde{n p} \tilde{p}^{\prime \prime}$, respectively, the singly-smoothed and doubly-smoothed normalized mean price estimates, as follows:

$$
\tilde{\mathrm{np}}_{d-1}=2 \tilde{\mathrm{np}}_{d-1}^{\prime}-\tilde{\mathrm{np}}_{d-1}^{\prime \prime}
$$

where

$$
\begin{aligned}
& \widetilde{\mathrm{np}}_{d-1}^{\prime}=\beta \cdot \widetilde{\mathrm{np}}_{d-1}+(1-\beta) \cdot \widetilde{\mathrm{np}}_{d-2}^{\prime} \\
& \tilde{\mathrm{np}}_{d-1}^{\prime \prime}=\beta \cdot \tilde{\mathrm{np}}_{d-1}^{\prime}+(1-\beta) \cdot \widetilde{\mathrm{np}}_{d-2}^{\prime \prime}
\end{aligned}
$$

The model can be initialized simply by setting both smoothed series equal to the observed value at $d=1$. The parameter $\beta \in(0,1)$ provides computational stability in prediction between the two exponentially smoothed time series. We determined the value of $\beta$ using a hill-climbing process to minimize prediction error over a set of historical data and selected $\beta=0.5$. We will show later in $\$ 5.1$ how we compute a smoothed mean price estimate in TAC SCM where the only information available is the minimum and maximum price for the previous day.

We then compute the smoothed price trend as

$$
\tilde{\operatorname{tr}}_{d-1}=\frac{\beta}{1-\beta} \cdot\left(\tilde{\mathrm{np}}_{d-1}^{\prime}-\tilde{\mathrm{np}}_{d-1}^{\prime \prime}\right)
$$

Using the trend and the previous day's smoothed mean price $\widetilde{n p}_{d-1}$, we predict the daily smoothed prices from the current day $d$ for each day $n$ over the horizon $h$ as

$$
\widehat{\mathrm{np}}_{d+n}=\tilde{\mathrm{np}}_{d-1}+(1+n) \cdot \tilde{\operatorname{tr}}_{d-1}, \quad \text { for } n=1, \ldots, h .
$$

The predicted prices, $\widehat{\mathrm{np}}_{d+n}$, over the planning horizon $h$ are used as input for the exponential smoother regime prediction, which is described next. In contrast, both Markov regime prediction methods (described later) only use the previous day's estimated price, $\widetilde{n p}_{d-1}$, as input and make predictions using Markov transition matrices that are computed from historical data.

3.2.2. Exponential Smoother Regime Prediction. The exponential smoother prediction process we described yields estimates of future mean prices but no information on price distributions. To obtain price distributions we translate the estimates of future prices to regime predictions and then we predict price distributions from regimes (see §3.3). As we shall see later in \$5.3.2, doing so actually improves price predictions as well.

Using the predicted mean price $\widehat{n p}_{d+n}$ computed with (8), we obtain the density of $\widehat{n p}_{d+n}$ dependent on regime $\hat{R}_{k}$ using (2), and the predicted probability of regime $\hat{R}_{k}$ dependent on the predicted normalized price $n$ days into the future, $\widehat{\mathrm{np}}_{d+n}$, using (3). Note that we use $\hat{R}_{k}$ to denote a particular predicted regime $R_{k}$.

Because the regime information is obtained from historical data, prices and corresponding regime probabilities can be computed in advance and stored in a table, reducing the subsequent real-time computations to a table lookup. This predictor is not as flexible as the others we will describe next, because it does not learn patterns in the data, but it is easy to compute. We use the term "exponential smoother with regimes" to describe this combination of using the exponential smoother to predict prices and then a table lookup to find the corresponding regime probabilities. 
3.2.3. Markov Regime Prediction. We model the short-term prediction of future regimes as a Markov prediction (Markov $P$ ) process. The prediction is based only on the most recent price $\widetilde{\mathrm{np}}_{d-1}$ and on historical data. We first compute a Markov transition matrix for regime transitions, $\mathbf{T}\left(r_{d+n} \mid r_{d-1}\right)$, by a counting process using historical data. This matrix represents the posterior probability of transitioning from regime $r_{d-1}$ on day $d-1$ to regime $r_{d+n}$ on day $d+n$, where $r=R_{k}$ for $k=1, \ldots, M$, and $M$ is the number of regimes. We use $\vec{P}\left(\hat{r}_{d-1} \mid \tilde{\mathrm{np}}_{d-1}\right)$ to indicate a $M$-dimensional vector of the posterior probabilities of the predicted regimes $\hat{r}$ on day $d-1$.

We further distinguish between two types of Markov predictions: (1) n-day and (2) repeated oneday prediction. An $n$-day prediction computes a transition matrix for each of the $n$ days in the future and multiplies these matrices by the current day regime estimates to predict regimes $n$ days in the future. The repeated one-day matrix instead assumes a stable transition matrix and multiplies itself $n$ times to produce the transition probabilities for $n$ days in the future.

The prediction of the posterior distribution of regimes $n$ days into the future, $\vec{P}\left(\hat{r}_{d+n} \mid \tilde{\mathrm{np}}_{d-1}\right)$, is done recursively as follows:

1. n-day prediction. The n-day prediction is based on training a separate Markov transition matrix for each day in the planning horizon $h$; i.e., $\mathbf{T}_{n}\left(r_{d+n} \mid r_{d-1}\right)$, for $n=1, \ldots, h$.

$$
\begin{array}{r}
\vec{P}\left(\hat{r}_{d+n} \mid \tilde{\mathrm{np}}_{d-1}\right)=\mathbf{T}_{n}\left(r_{d+n} \mid r_{d-1}\right) \vec{P}\left(\hat{r}_{d-1} \mid \tilde{\mathrm{np}}_{d-1}\right) \\
\text { for } n=1, \ldots, h .
\end{array}
$$

2. Repeated one-day prediction. The repeated oneday prediction is done by using the one-day prediction matrix $\mathbf{T}_{1}\left(r_{d} \mid r_{d-1}\right)$ multiple times.

$$
\begin{array}{r}
\vec{P}\left(\hat{r}_{d+n} \mid \tilde{\mathrm{np}}_{d-1}\right)=\prod^{n} \mathbf{T}_{1}\left(r_{d} \mid r_{d-1}\right) \vec{P}\left(\hat{r}_{d-1} \mid \widetilde{n p}_{d-1}\right) \\
\qquad \text { for } n=1, \ldots, h .
\end{array}
$$

In a completely stable environment, $n$ repeated one-day Markov predictions would lead to the same results as a single application of the appropriate $n$-day prediction. In real environments, however, this assumption is often violated because the environment changes dynamically over time. When making predictions far in the future, the repeated one-day method reaches a stationary distribution where all transition probabilities converge to the same values. This drawback can be avoided by using an $n$-day Markov prediction matrix. Details can be found in the online appendix.

The prior regime probability for the first day needs to be assigned according to the market situation. For instance, in TAC SCM we set the prior regime probability for the first day to $100 \%$ extreme scarcity, to represent the condition when the initial finished product inventories are zero.

3.2.4. Markov Regime Correction-Prediction. For long-term prediction of future regimes, we use a Markov correction-prediction (Markov $C-P$ ) process, where the prediction part is similar to the Markov prediction described above but taking into account the entire real-time price history, $\tilde{\mathrm{np}}_{1}, \ldots, \widetilde{\mathrm{np}}_{d-1}$, instead of a single day $\tilde{n p}_{d-1}$. A Markov correction-prediction process is better when the process depends on realtime transitions in the immediate past beyond a single day. Both Markov $P$ and Markov $C-P$ processes depend on either one-day or $n$-day transition matrices, which are learned offline from historical data. The Markov C-P method is based on two distinct operations done in sequence:

1. A correction (recursive Bayesian update) of the posterior probabilities of the regimes based on the history of prices starting from the first, $\tilde{n p}_{1}$, until the most recent on day $d-1$ is given by

$$
\begin{aligned}
& \vec{P}\left(\hat{r}_{d-1} \mid\left\{\tilde{\mathrm{np}}_{1}, \ldots, \tilde{\mathrm{np}}_{d-1}\right\}\right) \\
& =\frac{\vec{P}\left(\tilde{\mathrm{np}}_{d-1} \mid \hat{r}_{d-1}\right) \vec{P}\left(\hat{r}_{d-1} \mid\left\{\tilde{\mathrm{np}}_{1}, \ldots, \tilde{\mathrm{np}}_{d-2}\right\}\right)}{\sum_{r_{d-1}=1}^{M} \vec{P}\left(\tilde{\mathrm{np}}_{d-1} \mid r_{d-1}\right) \vec{P}\left(r_{d-1} \mid\left\{\tilde{\mathrm{np}}_{1}, \ldots, \tilde{\mathrm{np}}_{d-2}\right\}\right)} .
\end{aligned}
$$

2. A prediction of the posterior probabilities of regimes $n$ days into the future, $\vec{P}\left(\hat{r}_{d+n} \mid\left\{\tilde{n p}_{1}, \ldots, \tilde{n p}_{d-1}\right\}\right)$, is done recursively as in the Markov prediction case. The $n$-day prediction is given by

$$
\begin{array}{r}
\vec{P}\left(\hat{r}_{d+n} \mid\left\{\tilde{n p}_{1}, \ldots, \tilde{\mathrm{np}}_{d-1}\right\}\right) \\
=\mathbf{T}_{n}\left(r_{d+n} \mid r_{d-1}\right) \vec{P}\left(\hat{r}_{d-1} \mid\left\{\tilde{\mathbf{n p}}_{1}, \ldots, \tilde{\mathrm{np}}_{d-1}\right\}\right) \\
\text { for } n=1, \ldots, h .
\end{array}
$$

The repeated one-day prediction is given by

$$
\begin{array}{r}
\vec{P}\left(\hat{r}_{d+n} \mid\left\{\tilde{n p}_{1}, \ldots, \tilde{\mathrm{np}}_{d-1}\right\}\right) \\
=\prod^{n} \mathbf{T}_{1}\left(r_{d} \mid r_{d-1}\right) \vec{P}\left(\hat{r}_{d-1} \mid\left\{\tilde{\mathrm{np}}_{1}, \ldots, \tilde{\mathrm{np}}_{d-1}\right\}\right) \\
\text { for } n=1, \ldots, h .
\end{array}
$$

Note that Equations (12) and (13) use a matrix multiplication, whereas Equation (11) uses an element wise multiplication.

3.2.5. Computational Complexity of Economic Regimes. The key computational requirements of the regime model's price predictions involve propagating the hidden state density forward, called forward filtering. Forward filtering has well-known computational complexity results, with time complexity of $O\left(M^{2} T\right)$ and memory complexity of $O(M T)$ (Khreich et al. 2010), where $M$ is the number of regimes, and $T$ is the 
number of time steps used for making a prediction. For our Markov $P$ process $T$ is the number of forecast steps, and for the Markov C-P process it is the entire history plus the number of extrapolated steps. The dependence on time reflects that the algorithm takes the entire history of the sequence into account when making predictions, whereas the quadratic dependence on the regime state size is because of the matrix multiplication used to propagate regime state probabilities. These worst case results can potentially be improved by limiting the data history the algorithm processes before making predictions, which would make regime prediction's complexity results equivalent to exponential smoothing. Exponential smoothing has memory and time complexity $O(1)$ because the algorithm only needs a fixed finite amount of previous data to make predictions.

\subsection{Price Distribution and Order Probability Prediction}

Using the predicted regime distribution, we can now compute the predicted price distribution ${ }^{2}$ as follows:

$$
\begin{aligned}
p & \left(\widehat{\mathrm{np}}_{d+n} \mid \widetilde{\mathrm{np}}_{d-1}\right) \\
& =\sum_{i=1}^{M} p\left(\mathrm{np} \mid R_{i}\right) P\left(\hat{R}_{i, d+n} \mid \widetilde{\mathrm{np}}_{d-1}\right) \\
& =\sum_{j=1}^{N} \sum_{i=1}^{M} \underbrace{P\left(\zeta_{j} \mid R_{i}\right) P\left(\hat{R}_{i, d+n} \mid \widetilde{\mathrm{np}}_{d-1}\right)}_{P\left(\zeta_{j, d+n}\right)} p\left(\mathrm{np} \mid \zeta_{j}\right) \\
& =\sum_{j=1}^{N} P\left(\zeta_{j, d+n}\right) p\left(\mathrm{np} \mid \zeta_{j}\right), \quad \text { for } n=1, \ldots, h,
\end{aligned}
$$

where $\widehat{n p}_{d+n}$ is the predicted normalized price on day $d+n, P\left(\hat{R}_{i, d+n} \mid \tilde{\mathrm{np}}_{d-1}\right)$ is an element of the predicted regime probability vector given by (9) or by (10), and again $M$ is the number of regimes and $N$ the number of Gaussians. After marginalizing over the regimes we obtain new priors for the individual Gaussians $\zeta_{j}$ in the GMM. To obtain the predicted price distribution, we sample the updated model every day over the planning horizon $h$ with values over the whole range of $\mathrm{np}$. A detailed example for our testbed is presented in $§ 5.2$.

From the predicted price distribution we can compute the predicted normalized price $\widehat{\mathrm{np}}_{d}$ for day $d$ as the median of the distribution. We can also use the predicted distribution to construct the cumulative density function $\operatorname{CDF}(n p)$ for normalized price $n p$. Given $\mathrm{CDF}(\mathrm{np})$, the probability of a customer order, $P($ order $\mid \mathrm{np})$, can be computed as $P($ order $\mid \mathrm{np})=1$ $\mathrm{CDF}(\mathrm{np})=1-\int_{0}^{\mathrm{np}} p\left(\mathrm{np}^{\prime}\right) d \mathrm{np}^{\prime}$.

${ }^{2}$ We describe this using Markov prediction, but a similar equation holds for the other prediction methods.

\subsection{Using Economic Regimes for Strategic and Tactical Decisions}

We now discuss an approach that takes advantage of our prediction models to maximize expected profit over some period in the future. An agent or human decision maker making sales decisions in markets that are affected by price fluctuation needs to make two broad decisions: (1) whether to sell or hold inventory and (2) if the decision is to sell at least part of the inventory, what price should it quote. Holding inventory makes sense when higher prices are expected in the future. At the other extreme, if the firm is holding a large inventory and the future economic outlook looks bleak, it should sell down inventory to liquidate it. The decision to hold a certain level of inventory for the future is a strategic decision, and setting the price for the current time period is a tactical decision.

3.4.1. Strategic Decision-Resource Allocation. We first focus on a common set of information that is typically available in a manufacturing environment:

$-\mathscr{C}$ is the set of all available component types. Each component $c$ is needed to produce some subset of products $\mathscr{G}_{c}$.

- $G$ is the set of all products that can be manufactured and sold. Each product's components are represented by the set $\mathscr{C}_{g}$.

-For a day $d$ within a planning horizon $h$, expected customer demand is represented by a set $\mathbb{Q}_{d}$ of customer requests for quotes. We assume customers ask for prices and will buy at the lowest quoted price. Each $q \in \mathscr{Q}_{d}$ specifies a product type $g_{q}$, a lead time of $i_{q}$ days, a volume $v_{q}$, and a reserve price $\rho_{q}$.

-For a day $d$ within the planning horizon $h$, the agent expects to have an inventory of raw materials $I_{d, c}$ for each component type $c \in \mathscr{C}$ and an inventory of finished goods consisting of $I_{d, g}$ for each type of good $g \in \mathscr{G}$.

- On any given day $d$, there is an unsold inventory $I_{d, g}^{\prime}$ of good $g$ and an expected uncommitted inventory $I_{d, c}^{\prime}$ of parts of type $c$. This includes parts in current inventory and parts that are expected to be delivered by day $d$ and excludes parts that are allocated to produce goods for outstanding customer orders.

On day $d$, the total demand $D_{d, g}$ for a given good $g$ among $Q_{d}$ is the total of the requested quantities among requests for good $g, D_{d, g}=\sum_{q \in Q_{d}} v_{q}$. The effective demand $D_{d, g}^{\text {eff }}\left(\right.$ price $\left._{d, g}\right)$ is the portion of total demand with reserve prices $\rho_{g} \geq$ price $_{d, g}$. Note that for computing effective demand and sales quantities we must use non-normalized price rather than normalized price $\mathrm{np}$.

Our goal is to choose a sales quantity $A_{d, g}$ for each product $g$ over each day of the planning horizon $h$ to maximize expected profit $\Phi=\sum_{d=0}^{h} \sum_{g \in \mathscr{G}} \Phi_{d, g} A_{d, g}$, 
where $\Phi_{d, g}$ is the discounted profit for day $d$ and $A_{d, g}$ is the quantity of product the agent wishes to sell for good $g$ on day $d$. The discounted profit is computed as

$$
\Phi_{d, g}=\gamma_{d}\left(\text { price }_{d, g}-\operatorname{cost}\left(\mathscr{C}_{g}\right)\right) \text {, }
$$

where $\gamma_{d}$ is a discount term that can be seen as a rough approximation of inventory holding and opportunity costs. It can also be used to encourage early selling, as a hedge against future uncertainty. The

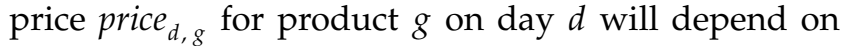
the demand $D_{d, g}$ and the quantity of product $A_{d, g}$ we wish to sell, as well as other factors that we will discuss in §3.4.2.

We assume the daily production capacity is $F$, each unit of good $g$ requires $y_{g}$ production cycles, and $F_{m}^{\text {commit }}$ is the factory capacity that is committed to manufacture outstanding customer orders that are due on or before a day $m$ days in the future and are not satisfiable by existing finished goods inventory. Now we can define an optimization problem that maximizes total profit $\Phi$ by choosing appropriate sales quotas $A_{d, g}$ :

$$
\begin{array}{ll}
\max & \Phi=\sum_{d=0}^{h} \sum_{g \in \mathscr{G}} \Phi_{d, g} A_{d, g} \\
\text { subject to } & \forall d, \forall g, A_{d, g}<D_{d, g}^{\mathrm{eff}} \\
& \forall m \in 0 \ldots h, \forall c \in \mathscr{C}, \\
& \sum_{d=0}^{m} \sum_{g \in \mathscr{G}_{c}} A_{d, g} \leq I_{m, c}^{\prime}+\sum_{g \in \mathscr{S}_{c}} I_{m, g}^{\prime} \\
\forall n \in 0 \ldots h, \\
\sum_{g \in \mathscr{G}} y_{g}\left(\sum_{d=0}^{n} A_{d, g}-I_{d, g}^{\prime}\right) \leq n F-F_{n}^{\mathrm{commit}} .
\end{array}
$$

Equation (17) is the demand constraint. Equation (18) is the supply constraint over the planning horizon, $h$, that restricts maximum supply that can be created using the parts and the finished goods in existing inventory. This may be conservative because we are considering goods or their parts to be available at the time we propose to sell them, not when we expect to ship them. The constraint also ensures that every subset of product types that can share some component is not overcommitted. Equation (19) is the manufacturing constraint that restricts the sales quantity to what is in the unsold inventory or can be manufactured within the planning horizon.

To appropriately choose sales quotas $A_{d, g}$, we need to set prices. For instance, in \$5.2, we describe several methods we use in TAC SCM to estimate price distributions, which can in turn be used to estimate $P$ (order $\mid$ price $)$ as described in the next section.
Because the quantity we expect to sell is just the effective demand multiplied by the order probability at the price we set, we can then express $A_{d, g}$ as

$$
A_{d, g}=P\left(\text { order } \mid \text { price }_{d, g}\right) D_{d, g}^{\text {eff }}\left(\text { price }_{d, g}\right) \text {. }
$$

Combining (15) with (20), the objective function (16) becomes

$$
\begin{aligned}
\max \Phi= & \left.\sum_{d=0}^{h} \sum_{g \in \mathscr{G}} \gamma_{d} \text { price }_{d, g}-\cos t\left(\mathscr{C}_{g}\right)\right) \\
& \cdot P\left(\text { order } \mid \text { price }_{d, g}\right) D_{d, g}^{\mathrm{eff}}\left(\text { price }_{d, g}\right) .
\end{aligned}
$$

Note that even if we assume the order probability and effective demand are linear, (21) is at least cubic in price $_{d, g}$. Because (21) is probably unsolvable in real time, we focus on developing heuristics that can be embedded in automated agents. An obvious simplification is to assume that the partial derivative of the order probability function with respect to price is large, much larger than the partial derivative of profit with respect to price. This is equivalent to saying that (most) sales occur very close to a "market clearing price." Then per-unit profit and effective demand can be computed separately by substituting an estimated clearing price price clear for the actual sales price into (21). ${ }^{3}$ We will show how to compute the clearing price price clear in the next section.

3.4.2. Tactical Decision-Sales Offer Pricing. Once the strategic sales process has determined daily sales quotas, we must set prices that will move those quotas in expectation. This amounts to finding, for each good, the value for price $_{d, g}$ that satisfies (20). We call this price offer $e_{d, g}$, and we estimate it by first estimating the market clearing price price ${ }_{d, g}^{\text {clear }}$ and using it to locate the predicted order-probability distribution $P$ (order $\mid$ price) as described in §3.3. The clearing price for the current day is estimated by combining the observed price (from the Price monitor module in Figure 3) with an offset $\delta_{d, g}$ that is computed by observing the market's response to our offers, as follows.

We compute price ${ }_{d, g}^{\text {offer }}$ by choosing a target order probability $P^{\text {offer }}=A_{d, g} / D_{d, g}^{\text {eff }}\left(\right.$ price $\left._{d, g}^{\text {clear }}\right)$ and finding the corresponding offer price price $e_{d, g}^{\text {offer }}$ from (20) by solving $P^{\text {offer }}=P\left(\right.$ order $\mid$ price $\left._{d, g}^{\text {offer }}\right)$. Assuming the market clears once each day, the order volume $O_{d, g}$ is the number of orders placed for good $g$ in response to our offers on the previous day. Market response to pricing decisions is stochastic, so the number of orders received may be higher or lower than our expected sales $A_{d, g}$. We then compute a price that reflects the actual number of

\footnotetext{
${ }^{3}$ This assumption can be partially relaxed by breaking sales price distributions into discrete "chunks" with separate demand constraints.
} 
orders price $e_{d-1, g}^{\text {order }}$ for the previous day by computing a point $P^{\text {order }}=O_{d, g} / D_{d-1, g}^{\text {eff }}\left(\right.$ price $\left._{d-1, g}^{\text {clear }}\right)$ on an adjusted probability curve $P^{\prime}$ (order $\mid$ price), obtained by translating the original order probability function to pass through the point (price $e_{d, g}^{\text {offer }}, O_{d, g} / D_{d-1, g}^{\text {eff }}$ ). We then use the translated probability function $P^{\prime}$ (order $\mid$ price) to compute price $_{d-1, g}^{\text {order }}$, as visualized in Figure 1.

The difference $\quad$ diff $_{d-1, g}=$ price $_{d-1, g}^{\text {order }}-$ price $_{d-1, g}^{\text {offer }}$ is then used each day to compute price clear $=$ price $_{d, g}^{\text {pred }}+\delta_{d, g}$ where price $d_{d, g}^{\text {pred }}$ is the predicted market price for product $g$, the unnormalized version of the predicted mean price from (22), and $\delta_{d, g}$ is updated daily using simple exponential smoothing as $\delta_{d, g}=\alpha \delta_{d-1, g}+(1-\alpha)$ diff $f_{d-1, g}$ for some appropriate value of $\alpha \in[0,1]$.

\subsubsection{Computational Complexity of Resource} Allocation. An efficient algorithm for linear programming is described by Karmarkar (1984). It has a worstcase computational complexity of $O\left(X^{3.5} L\right)$, where $X$ is the number of variables in the objective function, and $L$ is a function of the desired numerical accuracy. The complexity is polynomial; the average case complexity is typically much lower. The problem size for our problem is also polynomial, dominated by inventory constraints. With 16 products and a 20-period planning horizon, we have 320 variables; a typical situation generates $15,000-30,000$ constraints. The maximum number of constraints is quadratic in the planning horizon and in the average number of components making up a product (4 in our case study), and it is linear in the number of components in the catalog (10 in our case study) and in the number of products that share a component (which in our case study ranges from 2 to 8 ). The actual number of rows is typically less than $20 \%$ of the maximum because we discard rows that do not add constraint.

Figure 1 Estimating Market Price, Given Order Volume 0 , Sales Quota $A$, Effective Demand $D^{\text {eff }}$, and an Order Probability Function $P$ for Each Day and Each Product

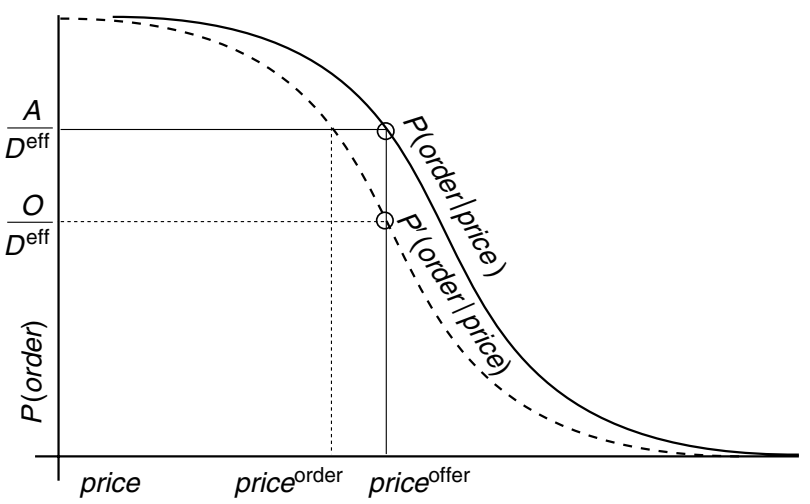

For our experiments in TAC SCM we have used lp-solve, ${ }^{4}$ which is less efficient than the Karmarkar algorithm. On a modern $3 \mathrm{GHz}$ 32-bit PC, the typical solution time is less than one second, and we have not exceeded eight seconds in more than 100,000 runs.

\section{A Case Study: The Trading Agent Competition for Supply Chain Management (TAC SCM)}

We have implemented and tested our approach in an agent-based simulated market environment (Swaminathan et al. 1998) in which agents must compete with each other in both procurement and sales markets while simultaneously managing inventories, fulfillment, and a manufacturing process. The annual Trading Agent Competition for Supply Chain Management (Collins et al. 2005, 2010b) is a competitive agent-based simulation of an abstract supply chain environment, where software agents make all the decisions. TAC SCM simulates a market where six autonomous agents compete to maximize profits over a one-year life cycle for a set of computer models. The simulation takes place over 220 virtual days, each lasting 15 seconds of real time, of which about 12 seconds can be used for computation and the rest are needed for communication and simulation server overhead. TAC SCM agents earn money by selling computers they assemble using parts that they must competitively acquire from suppliers. Each agent has a finite manufacturing capacity to allocate across a set of products. Each agent must pay to store raw materials and finished-product inventory and must borrow money to build its initial inventory. The agent with the highest bank balance at the end of the simulation wins. TAC SCM is an abstract model of real markets, leaving out many factors such as quality of products, marketing strategies, long-term procurement contracts, transportation costs, etc., but has the advantage of enabling a systematic comparison of different strategies and approaches.

Each agent in TAC SCM can produce 16 different types of products, categorized into three market segments (low, medium, and high quality products). Demand in each market segment varies randomly during the simulation. Every day each agent receives a set of requests for quotes (RFQs) from several potential customers. Each customer RFQ specifies the type of product requested, along with quantity, due date, reserve price, and penalty for late delivery. Each agent may choose to bid on some or all of the day's RFQs. Customers accept the lowest bid that is at or below their reserve price and notify the winning agent. The agent must ship customer orders on time or pay a

\footnotetext{
${ }^{4} \mathrm{http}$ ///sourceforge.net/projects/lpsolve
} 


\section{Figure 2 TAC SCM Scenario}

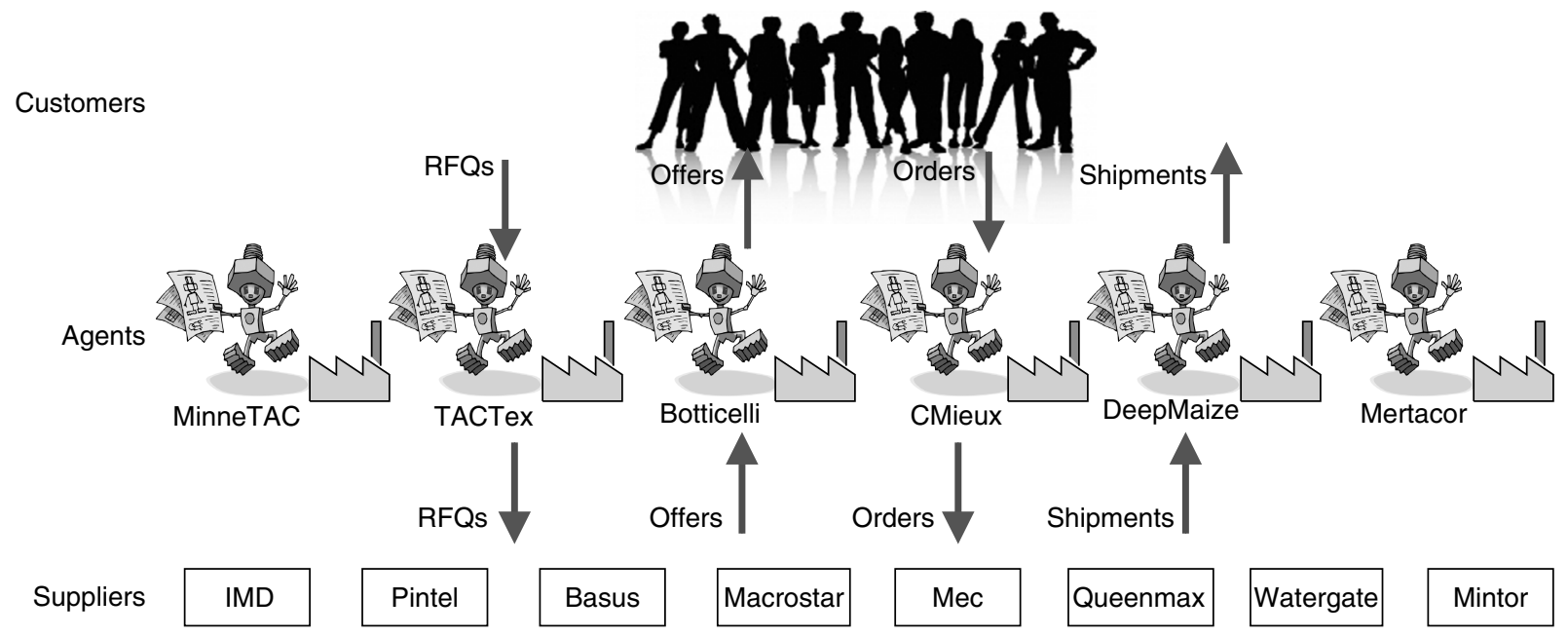

penalty for each day an order is late. Because the environment is a competitive oligopolistic market, actions of each agent significantly affect the markets and hence other agents' profits and strategies.

Organized competitions, such as TAC SCM (Collins et al. 2010b) and Power TAC (Ketter et al. 2011), along with many related computational tools are driving research into a range of interesting and complex domains that are both socially and economically important (Bichler et al. 2010). Because such experimental platforms allow market structures to be evaluated under a variety of real-world conditions and competitive pressures, they can also be used to effectively uncover potential hazards of proposed market designs in the face of strategic behaviors on the part of the participating agents. This can help policy makers in policy and regulation design. For instance, opportunities for agents to manipulate the TAC SCM competition in unintended ways were uncovered (Ketter et al. 2004), and the simulation model was subsequently updated to more accurately model realistic supplier behavior.

\subsection{Price Prediction in TAC SCM}

Typical approaches used for price forecasting in TAC SCM are exponential smoothing and linear regression methods (Benisch et al. 2006, Chatzidimitriou et al. 2008, Jordan et al. 2007, Podobnik et al. 2008). Some researchers (Zhang et al. 2004) have applied a game theoretic approach to set offer prices, using a variation of the Cournot game for modeling the product market. Others (He et al. 2006) use fuzzy reasoning to set offer prices. The TacTex agent predicts the distribution of prices using a weighted average of uniform densities between the low and high prices from the previous five days and predicts into the future by assuming that the distribution of prices does not change (Pardoe and Stone 2006). The Deep Maize agent uses a $k$-nearest-neighbors approach to map current observable indicators (prices, demand, etc.) to learned distributions of current and future prices. It employs both offline and online learning that optimizes predictions according to a logarithmic scoring rule. Deep Maize uses tournament and self-play data and combines them using an affine transformation (Kiekintveld et al. 2009). It determines the parameters of the affine transformation by a brute-force search to find values that minimize the scoring rule. The most recent version of TacTex uses different machine learning techniques, such as particle filtering, applied to historical data and online adaptation to predict current and future prices (Pardoe and Stone 2007). Finally, the Mertacor agent uses a decision tree machine learning technique to predict current and future prices (Chatzidimitriou and Symeonidis 2009).

In competitive oligopolistic markets with dynamic pricing, such as TAC SCM, it is also important to model "order probability" - the probability of winning a customer order at a given price. In TAC SCM, this probability is typically either estimated by linear interpolation from the minimum and maximum daily prices (Pardoe and Stone 2004), using a linear cumulative density function (CDF; Benisch et al. 2004) to estimate the relationship between offer price and order probability, or using a reverse CDF and factors such as quantity and due date (Ketter et al. 2004). The first two approaches provide a rough approximation of the real order probability function; the last approach requires the agent to deal with sparse high dimensional matrices that have to be updated every day during the game. Our approach of economic regimes circumvents these problems by using only observed market prices and quantities.

\section{Evaluation in TAC SCM}

We have implemented our approach to drive sales decisions in an agent for the TAC SCM scenario in 
Figure 3 Integrating a Regime Model Into Agent Sales Decision Processing. Links Connecting the Components Affected by the Regime Model Are Dashed

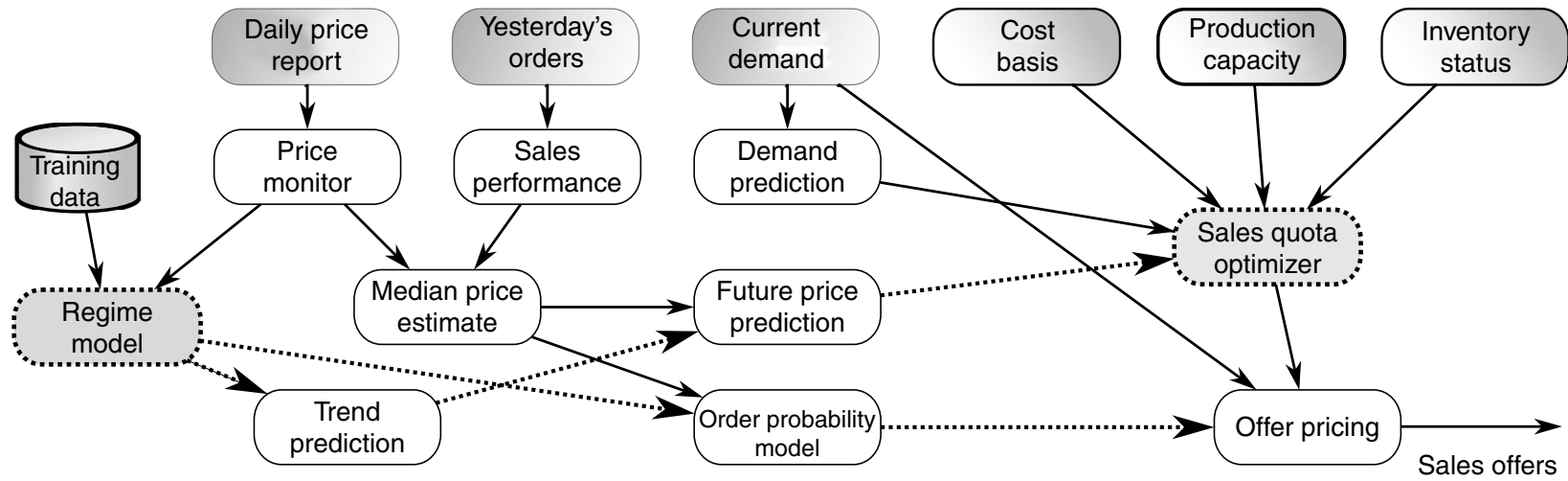

order to evaluate its performance. Our experimental agent uses a regime model to compute price distributions and price trends and to estimate order probability. Figure 3 shows a schematic view of the major elements the agent decision processing that leads to making offers at specific prices.

The key elements of this process are the regime model and its training data, described in $\$ 3$, and the sales quota optimizer, described in \$3.4.1. The final output is offer prices, computed as described in §3.4.2. Cost basis and inventory status information are derived from a procurement module, and production capacity data are produced by a production scheduling module.

\subsection{Real-Time Regime Identification in TAC SCM} In TAC SCM, agents are informed each day of the minimum and maximum order prices for each product on the previous day, but they cannot observe sales volume or the distribution of prices. As a crude approximation for the mean price one can use the mid-range normalized price, the price midway between the observed minimum and maximum. However, because observations of minimum and maximum prices are subject to noise, some of these observations may be outliers and not representative of the true price distribution.

Figure 4 (left) illustrates an example where daily mid-range prices do not always accurately estimate mean prices. The mean was computed after the simulation when all data are available. We observe a spike in the maximum price (especially on day 86, 87, 93, and 110) that biases the mid-range price. To lower the impact of sudden price changes, we smooth the minimum and maximum prices using a Brown linear exponential smoother (Brown et al. 1961) with $\beta=$ 0.5 to obtain the smoothed minimum $\tilde{n p}_{d-1}^{\min }$ and maximum $\tilde{\mathrm{pp}}_{d-1}^{\max }$ normalized prices, from which we compute the smoothed mid-range normalized price $\tilde{n p}_{d-1}$ as their average. With a slight abuse of notation in the description of TAC SCM, we use $\tilde{n p}_{d}$ for the midrange price instead of the mean price.

Figure 4 Min, Max, Mean, Mid-Range, and Smoothed Mid-Range Normalized Prices of Computers Sold Every Day in a Sample Run (Left); Real-Time Identification of Daily Regime Probabilities (Right)
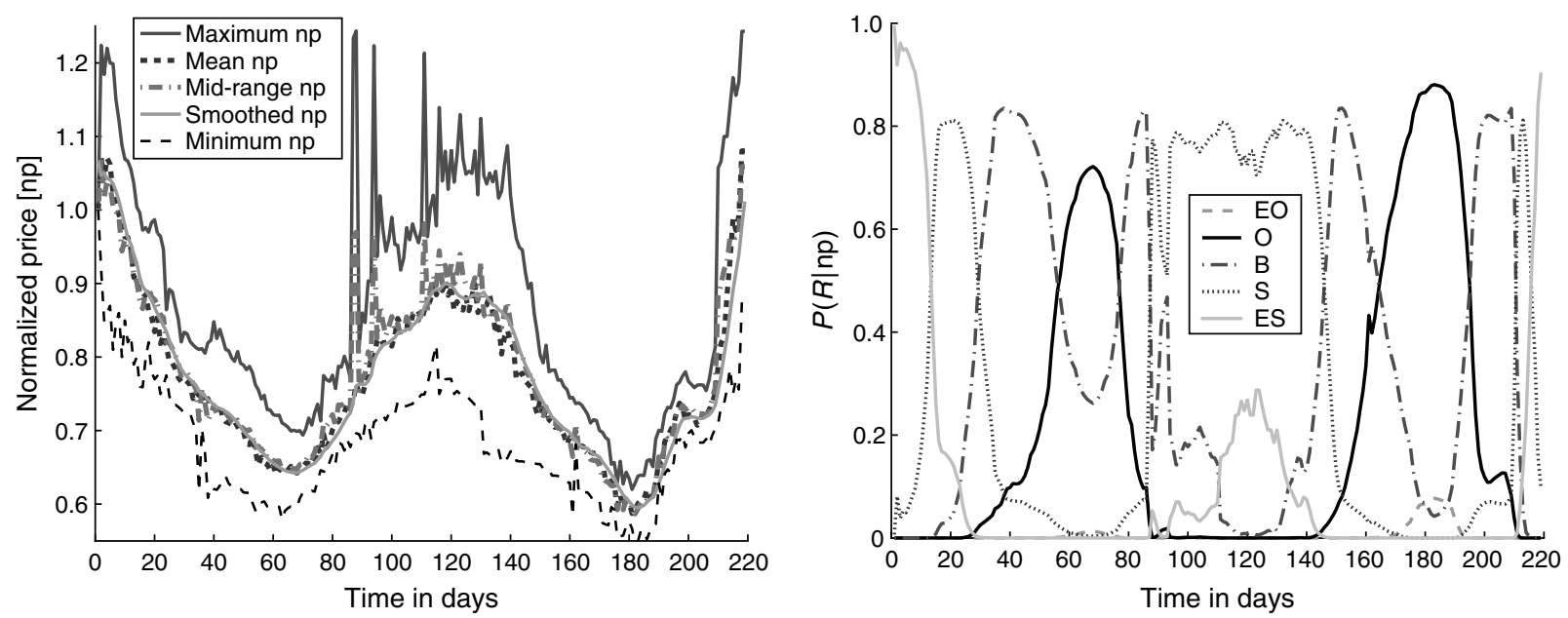
Figure 5 Predicted Price Density (Left) and Predicted Price Trend (PT) (Right) Using the Repeated One-Day Markov Matrix for Simulation 3,717@tac3 from Day 115 to Day 135

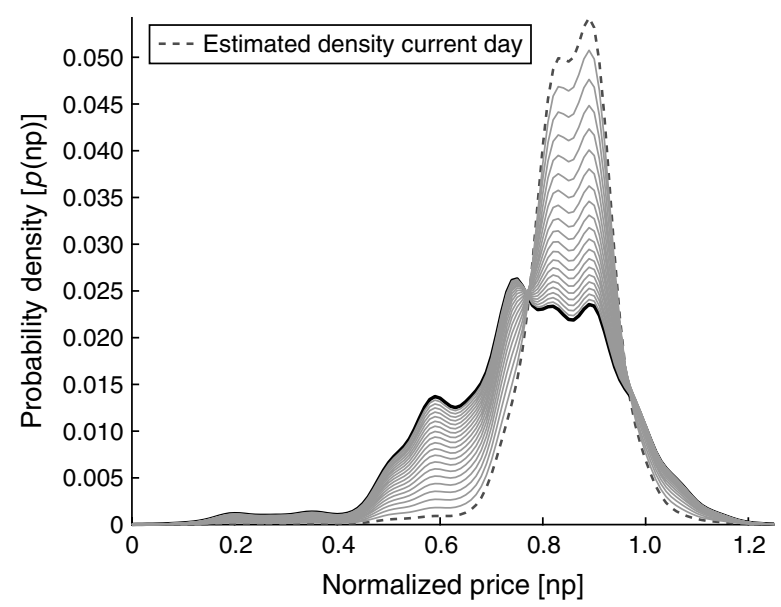

Figure 4 (right) shows the corresponding regime probabilities computed in real time during the simulation. The regimes are indicated as EO (Extreme Oversupply), O (Oversupply), B (Balanced), S (Scarcity), and ES (Extreme Scarcity). The graph shows that different regimes are dominant at different time points and that there are brief intervals during which two regimes are almost equally likely. We have reported a correlation analysis of the market parameters to regimes and more details on regime identification and other regime evaluation measures in Ketter et al. (2006), Ketter (2007), and Ketter et al. (2009).

\subsection{Prediction of Price Distribution and Trend}

To obtain a predicted price distribution we sample the price densities defined in (14) every day over the planning horizon $h$ with values for $\mathrm{np}$ between 0 and 1.25 because in TAC SCM reserve prices range up to $125 \%$ of nominal component prices. The samples are placed into $J=126$ price bins starting from $\mathrm{np}=0$ to $\mathrm{np}=1.25$ in 0.01 increments. Each bin $j$ contains the count of samples with the corresponding price, $\mathrm{np}(j)=(j-1) \cdot 0.01$. These counts are then normalized to obtain a probability. For instance, the mean of the distribution of the predicted normalized prices on day $d+n$ can be computed as

$$
\begin{array}{r}
E\left[\widehat{\mathrm{np}}_{d+n}\right]=\sum_{j=1}^{J} p\left(\widehat{\mathrm{np}}_{d+n}(j)=\mathrm{np}(j) \mid \tilde{\mathrm{np}}_{d-1}\right) \cdot \mathrm{np}(j), \\
\text { for } n=1, \ldots, h .
\end{array}
$$

To predict price trends we use also the $10 \%, 50 \%$, and $90 \%$ percentile of the predicted price distribution, which are interpolated from the discretized cumulative distribution.

Figure 5 (left) shows the forecast price density using the repeated one-day Markov matrix. The dashed

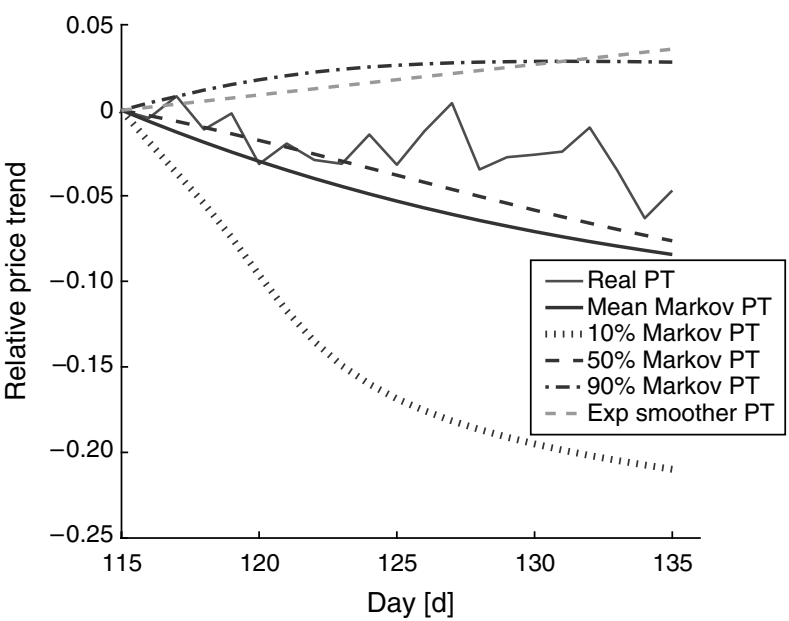

curve represents the price density for the first forecast day, the thick solid line shows the price density for the last forecast day, and the thin solid curves show the forecast for the intermediate days. As expected, the predicted price density broadens as we forecast further into the future, reflecting a decreasing certainty in the prediction. Figure 5 (right) shows the real mean price trend for this example along with forecast price trends, including the mean Markov prediction; the $10 \%, 50 \%$, and the $90 \%$ Markov density percentiles; and the exponential smoother.

Figure 6 (left) shows the forecast price density based on an $n$-day Markov prediction for the same simulation run presented above. We observe that the predicted price density shows significantly less variance as compared to using the repeated one-day Markov prediction. Figure 6 (right) shows the relative price trend for this example. The increased certainty in prediction is reflected by the reduced width of the probability envelope, represented by the $10 \%$ and $90 \%$ percentile contours. Note that the downward shift in actual prices, Figure 6 (right), is captured by the shift of the predicted future price distribution toward lower prices in Figure 6 (left).

The exponential smoother predictor in this example does not fare well ${ }^{5}$ because the exponential smoother puts too much weight on recently observed prices. In this case, prior to the prediction day the prices were increasing. The exponential smoother predictor takes the recent slope and extrapolates it into the future, whereas our Markov prediction method is able to learn patterns in the data and therefore does much better in predicting future changes.

${ }^{5}$ It is usually better than shown here for near-term predictions, but this example shows one of the advantages of our method. 
Figure $6 \quad$ Predicted Price Density (Left) and Predicted Price Trend (PT) (Right) Using the $n$-Day Markov Prediction for Simulation 3,717@tac3 from Day 115 to Day 135

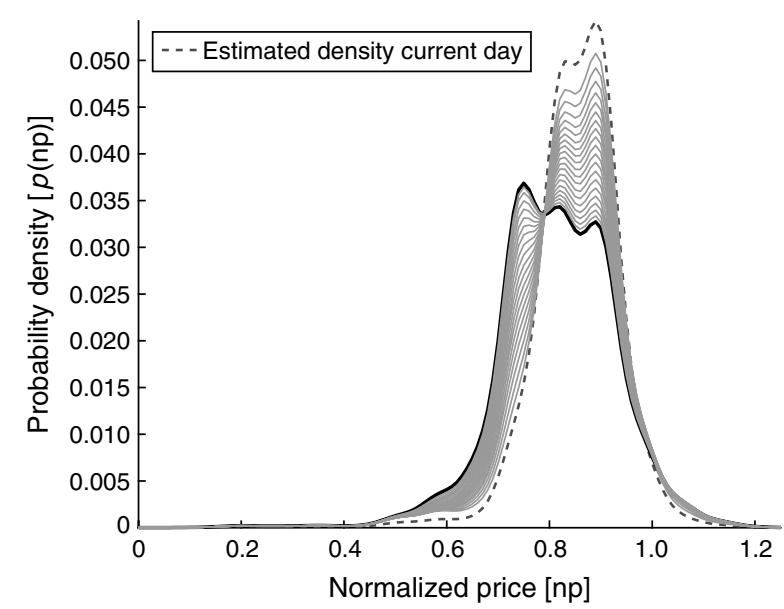

5.3. Prediction Accuracy

We now demonstrate the accuracy of the predictions made by our method by using it with historical data.

For our experiments, we used data from 28 runs, 18 used for training and 10 for testing (for details, please see the online appendix), played during the semifinals and finals of TAC SCM 2005. The mix of agents changed during the simulation runs, with a total of 12 agents in the semifinals and 6 in the finals. Because supply and demand vary in each market segment (low, medium, and high) independently of the other segments, our method is applied independently in each market segment.

5.3.1. Prediction of Regime Distribution. To determine how well the probability distribution of the predicted regime $\hat{R}$ matches the one of the actual regime $R$, we use the Kullback-Leibler (KL) divergence (Kullback and Leibler 1951, Kullback 1959). This measures the difference between two probability distributions in bits; smaller divergence values correspond to more accurate predictions. We calculate the KL divergence as

$$
\operatorname{KL}\left(\vec{P}_{\hat{R}} \| \vec{P}_{R}\right)=\sum_{i=1}^{M} \vec{P}_{\hat{R}}\left(r_{i}\right) \log \left(\frac{\vec{P}_{\hat{R}}\left(r_{i}\right)}{\vec{P}_{R}\left(r_{i}\right)}\right)
$$

by summing over the regimes $r_{i}$. The KL divergence can be interpreted in terms of how much additional data are needed to achieve optimal prediction performance. The precision of these data is given by the number of bits in the KL-divergence measure. For example a one bit difference would require an additional binary piece of information (Shannon 1948) such as "Were yesterday's bids all satisfied?" If the difference between two distributions is zero then the predictions are optimal in sense that the predicted and actual distributions match.

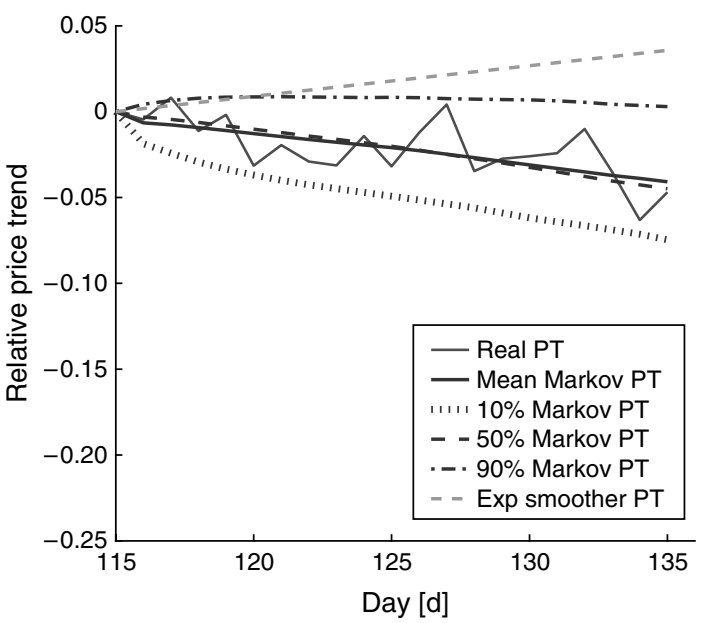

If the time-dependent distribution of a Markov process, in our case $\vec{P}_{\hat{R}}$, converges to a limit, $\vec{\Pi}=$ $\lim _{m \rightarrow \infty}\left\{\vec{P}_{\hat{R}}\right\}^{m}$ then $\vec{\Pi}$ is called the stationary distribution. When the stationary distribution exists it is characterized by the fix-point equation $\vec{\Pi}=\mathbf{T}_{n} \cdot \vec{\Pi}$. There are several ways to compute the stationary distribution, $\Pi$, which involve solving the eigenvalue problem specified in the above equation (for details consult the online appendix).

We introduced the $n$-day Markov matrix because we hypothesized that the $n$-day Markov matrix will take longer to reach the stationary distribution of its Markov process than the one-day Markov matrix, and therefore it will deliver a better prediction performance. We prove this hypothesis empirically by calculating the stationary distribution $\vec{\Pi}$ for the one-day and each n-day Markov matrices and comparing it with the Markov predicted regime distribution, using again the KL-divergence between $\vec{P}(\hat{R})$ and $\vec{\Pi}$.

In Figure 7 we show the KL-divergence for a GMM with 16 components and five regimes in the low market segment using a one-day Markov matrix (left) and an n-day Markov matrix (right) over the planning horizon. Points represent the KL-divergence between the Markov predicted regime distribution and the actual distribution, $\operatorname{KL}\left(\vec{P}_{\hat{R}_{\text {Markov }}} \| \vec{P}_{R}\right)$, and diamonds represent the KL-divergence between the double exponentially smoothed predicted distribution and the actual distribution $\operatorname{KL}\left(\vec{P}_{\hat{R}_{\text {Exp }}} \| \vec{P}_{R}\right)$. Pluses represent the KL-divergence between the Markov predicted regime distribution and the stationary distribution $\operatorname{KL}\left(\vec{P}_{\hat{R}_{\text {Markov }}} \| \vec{\Pi}\right)$. The figure shows that the one-day Markov matrix converges to the stationary distribution of the Markov process much faster than the $n$-day Markov matrices, as hypothesized.

The KL-divergence measures range from 0.28 bits (current day), to 0.80 bits (20 days), to 0.95 bits 
Figure 7 KL-Divergence Between Predicted, Actual, and Stationary Regime Distribution Using a Repeated One-Day (Left) vs. $n$-Day (Right) Markov Matrix, Computed Using Five Regimes on a GMM with 16 Components for the Low Market Segment Over the Testing Set

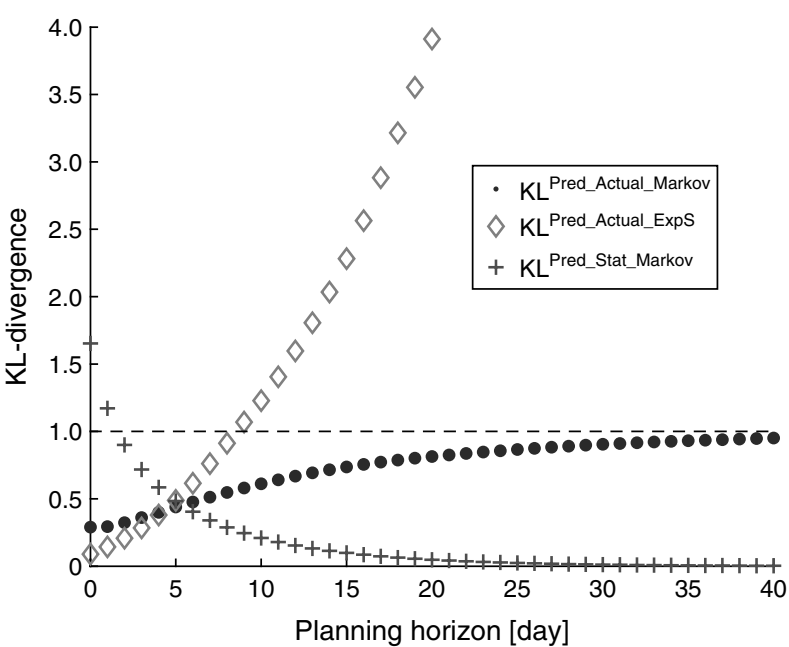

(40 days) of information when using the repeated oneday Markov matrix and from 0.28 bits (current day), to 0.66 bits ( 20 days), to 0.81 bits ( 40 days) of information when using the $n$-day Markov matrix, as opposed to the exponential smoother predictions, which range from 0.09 bits (current day), to 3.55 bits (20 days), to 12.62 bits (40 days). A KL-divergence less than or close to one is typically acceptable (Zhang and Cheung 2005), meaning that obtaining more information in the estimation procedure will not produce significant gains. We only show values of KL-divergences up to four because we want to highlight the differences for small values. The current day exponential smoother predictions are approximately 1.14 times better than the repeated one-day and $n$-day Markov predictions. On the other hand at 20 and 40 days, the exponential smoother predictions are approximately 6.73 and 3,259 times worse than the repeated one-day Markov predictions and 7.42 and 3,591 times worse than the $n$-day Markov predictions.

The KL-divergence values calculated using the $n$-day Markov matrix are always smaller than the repeated one-day Markov matrix, significantly so in the long term. This indicates a better fit between the predicted and the actual regime probabilities for the $n$-day Markov matrix. As a consequence the n-day Markov matrix should be used instead of the repeated one-day Markov matrix for strategic decision making. The best estimate for the short term (current day up to four days into the future) is given by the exponential smoother, which should be used to generate price densities for the short term and sales offer prices for the current day, i.e., for tactical decision making.

5.3.2. Comparison of Price Prediction Methods. We compute the price density, $p\left(\widehat{n p}_{d+n}\right){ }^{6}$ for the next

${ }^{6}$ For simplicity of notation here we leave out the dependence on historical normalized prices.

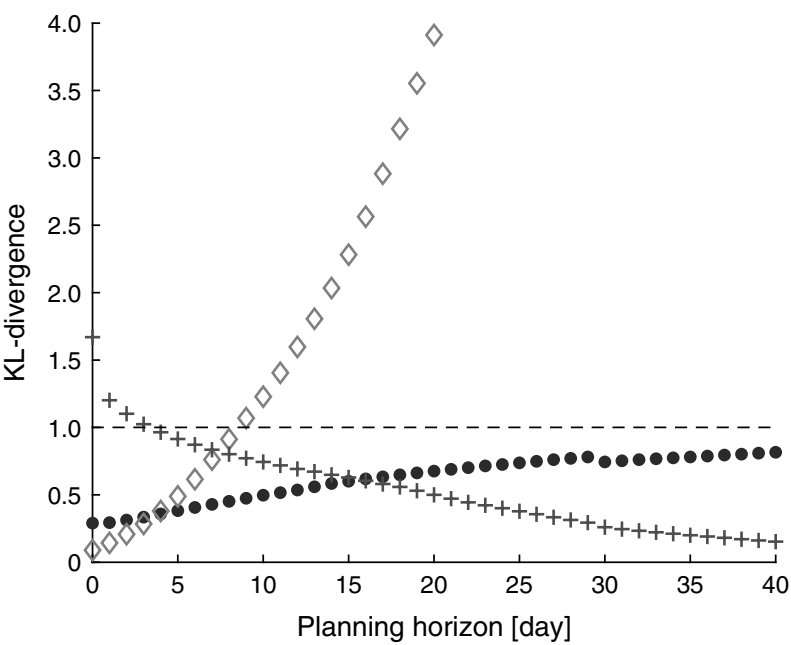

$n$ days into the future, where $p\left(\widehat{n p}_{d}\right)$ is the distribution of normalized prices on day $d$. We calculated the expected mean price using (22) and tracked different contours $(10 \%, 50 \%$, and $90 \%)$ of the price density curve. We calculated the root mean square error, $\operatorname{RMSE}\left(\widehat{n p}_{n}, \mathrm{np}_{n}\right)$, between the predicted normalized prices, $\widehat{\mathrm{np}}_{n}$, and the actual normalized price, $\mathrm{np}_{n}$, over a prediction interval of $n$ days in the planning horizon $h$, averaged across days and runs, to determine the accuracy of the price prediction as

$$
\operatorname{RMSE}\left(\overrightarrow{\mathrm{np}}_{n}, \overrightarrow{\mathrm{np}}_{n}\right)=\sqrt{\frac{\sum_{i=1}^{N_{G}} \sum_{d=1}^{N_{D}-n}\left(\overrightarrow{\mathrm{n}}_{d}^{n, i}-\overrightarrow{\mathrm{np}}_{d}^{n, i}\right)^{2}}{N_{G} \cdot\left(N_{D}-n\right)}},
$$

where $N_{D}$ is the number of days in a TAC SCM simulation, $N_{G}$ is the number of simulation runs, and $\overrightarrow{\mathrm{np}}_{d}^{n, i}$ is the predicted price vector for run $i$ for $n$ days into the future. In our experiments we chose a horizon $h=40$.

For these experiments we calculated the expected mean price using our three prediction methods, i.e., the exponential smoother with regimes (\$3.2.2), the Markov prediction (\$3.2.3), and the Markov correction-prediction (\$3.2.4) methods.

We have implemented four different comparison baselines, using approaches taken by successful TAC SCM agents.

1. The first baseline is an exponential smoother predictor (Wang et al. 2008). In TAC SCM, exponential smoothing and linear regression methods are also commonly used for price forecasting (Benisch et al. 2006, Kontogounis et al. 2006, Jordan et al. 2007, Podobnik et al. 2008).

2. The second is a constant predictor used by the Botticelli agent. The Botticelli predictor is based on 
Figure 8 RMS Error for Price Prediction Based on a Repeated One-Day (Left) vs. $n$-Day (Right) Markov Matrix; Three Regime-Based Prediction Methods Are Compared to Four Baseline Methods, Exponential Smoothing, ARIMA, and Methods Used by Other Successful TAC SCM Agents

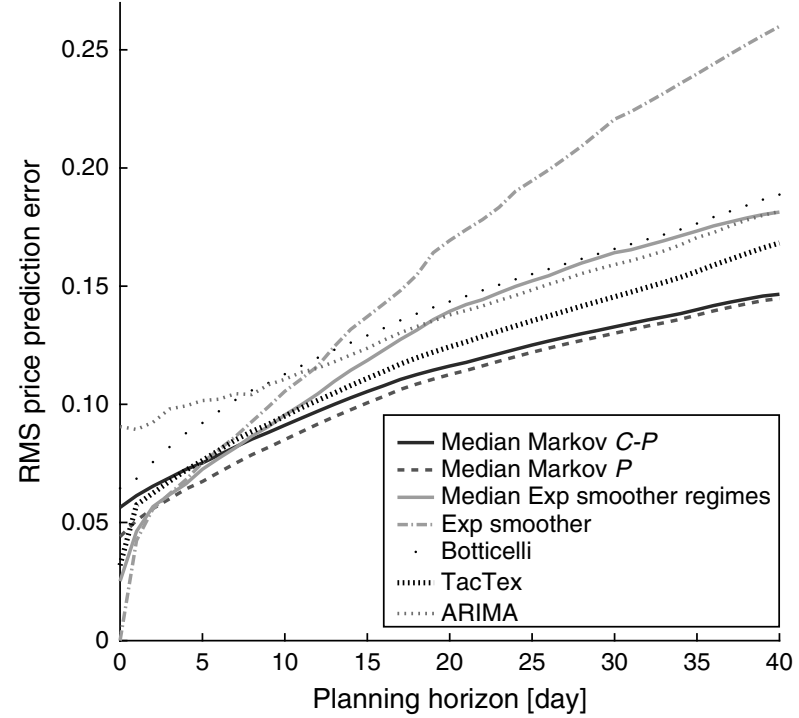

a daily evaluation of the relation between the ratio of offers won and an artificially constructed price variable. Each day, the predictor collects information about the ratio of offers won and the average offer price over the past $d$ days together with yesterday's minimum and maximum order price. The information is combined into a data set with $d+2$ observations and two variables, ratio of offers won and "price." The price variable consists of the $d$ average offer prices and the two extreme order prices of yesterday. The ratio of offers won is set equal to zero for yesterday's maximum price and equal to one for yesterday's minimum price. The predictor then regresses the ratio of offers won on the artificial price variable and uses the fitted model to induce the current mean price at a predicted offer probability of $50 \%$ (Benisch et al. 2004).

3. As a third baseline we implemented the heuristic predictor used by TacTex, the most successful agent of the TAC SCM tournament (Pardoe and Stone 2006). This method predicts prices using a weighted average of daily uniform densities between the low and high prices from the previous five days. We use weights of 0.3 for the two most recent days, 0.2 for the middle day, and 0.1 for the two oldest days. We predict future prices by assuming that the distribution of prices does not change. This method uses an information constraint in the current price level and relies completely on local price stability for predictive power. It was also used as a benchmark by the Deep Maize team to test their predictions (Kiekintveld et al. 2009).

4. The fourth is a standard time-series Auto-Regressive Integrated Moving Average (ARIMA) model (Box and Jenkins 1994, Enders 1995). After the identification and estimation processes, we arrived

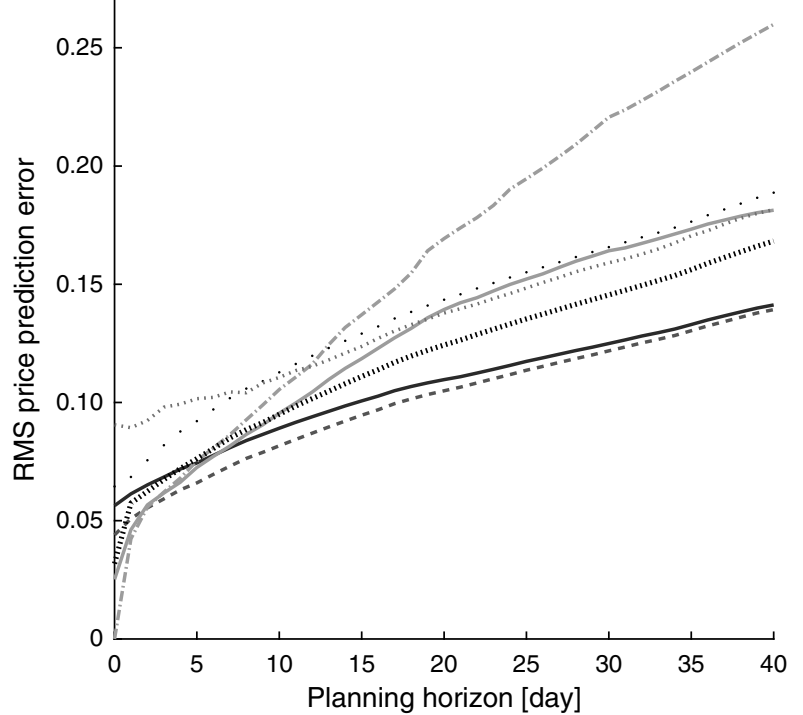

at an $\operatorname{ARIMA}(5,1,0)$ model, ${ }^{7}$ which we used for forecasting.

Figure 8 shows the RMS errors of our three predictors, i.e., the two Markov predictors using a repeated one-day matrix (left) versus the $n$-day matrix (right) and the exponential smoother with regime lookup, and compares them to the RMS errors of four baseline methods, i.e., a simple exponential smoother, the constant predictor used by Botticelli, the weighted average prediction technique used by TacTex, and the ARIMA model. An RMS error of 0.05 corresponds to an average prediction error of $4 \%$ and an RMS error of 0.25 corresponds to an average prediction error of $20 \%$. It is clear that the $n$-day Markov matrix improves the overall price prediction compared to the repeated one-day.

Results from our experiments show that although the exponential smoother performs reasonably well for short-term predictions, it is myopic, and even the simple modification where exponential smoothing utilizes regime information (described in \$3.2.2) improves performance. Further, for long-term predictions the Markov price predictors (described in $\$ \S 3.2 .3$ and 3.2.4) perform significantly better than not only the exponential smoother with regime information but also the constant predictor of Botticelli, the weighted average predictor of TacTex, and the ARIMA model. The TacTex predictor overall does well, even though not as well as the two Markov predictors that outperform all the other methods after the first few days. The ARIMA predictor shows inferior performance for

${ }^{7} \operatorname{ARIMA}(5,1,0)$ model parameters: $A R_{1,1}=0.46785, A R_{1,2}=$ $0.15953, A R_{1,3}=0, A R_{1,4}=0, A R_{1,5}=0.15488$, and $\mu=0.00131$. 
a planning horizon up to 10 days; after that its performance is comparable to the Botticelli predictor. For the first few days the simple exponential smoother predictor and the exponential smoother predictor with regime lookup outperform all other methods, but they do not work well for long term predictions, as we discussed earlier in \$3.2. The prices produced by both Markov $P$ and Markov $C-P$ are statistically similar to the observed prices because pairwise student $t$-tests failed to reject the null hypothesis of the equality of predicted $\widehat{\mathrm{np}}_{n}$ and actual observed prices $\mathrm{np}_{n}$ at $p=0.05$.

The differences in prediction accuracy between the baselines and the Markov regime predictions reflect exactly the advantage of the regimes-based price prediction methods over other alternative approaches. In general, we would expect a richer model, such as our regime model, to outperform a simpler model based on regression or time-series prediction. Our Markov prediction methods capture the rate of change (acceleration and deceleration) in the Markov transition matrices and therefore are able to predict price changes without having to assume a functional form, as nonlinear statistical models have to do. Another advantage of the regime model is that it has an intuitive qualitative interpretation, which can be used directly by either automated agents or human decision makers (Shmueli 2010).

The Markov C-P algorithm makes predictions using price data over many days in the preceding history. Implicitly it assumes that the price distribution follows a random walk, and thus its predictions are a compromise between the predictions based on any single previous day's prices. When the change in price is driven by short-term nonstationary trends, it may be better to base predictions only on the most recent price data because past prices could be unrepresentative of the systematic trends the market is undergoing. For example, if the prices are increasing each day for 10 days, prediction using the last day's price would be better. However, the Markov $C-P$ algorithm is likely to be better when price fluctuations are stochastic, as is the case in larger markets where no individual player makes a significant impact alone.

5.3.3. Prediction of Price Trends. Aside from daily prices, we assessed our ability to predict price trends because they play a crucial role in sales planning. We computed the estimated price trend $\widehat{\operatorname{tr}}_{d+n}$ for every day $n$ over the planning horizon $h$ as follows:

$$
\widehat{t r}_{n}=\operatorname{sgn}\left(\widehat{\mathrm{np}}_{d+n}-\widehat{\mathrm{np}}_{d}\right), \quad \text { for } n=1, \ldots, h,
$$

where sgn is the sign function, and $\widehat{n p}_{d}$ and $\widehat{n p}_{d+n}$ are the predicted prices on, respectively, day $d$ and day $d+n$. Because the agent has access only to the minimum and maximum prices of the previous day, it needs a one day forecast of the mid-range price to estimate the price on the current day $d$. If $\widehat{\operatorname{tr}}_{n}$ is positive, then the predicted prices are increasing; otherwise, they are decreasing.

Figure 9 displays the success rate of price trend sign prediction using a repeated one-day Markov matrix (left) and an n-day Markov matrix (right). Because the price trend is used for strategic decision making, we calculated the success rate starting at $d+5$. As the figure demonstrates, the Markov correctionprediction predicted the correct trend about $70 \%$ of the time and dominated the exponential smoothing approach. In general, the $n$-day Markov predictions performed better than the repeated one-day Markov matrix. In the figure we show the success rate using the expected means of the distributions, computed using Equation (22), as well as the medians of the distributions.

Figure 9 Success Rate of Price Trend Predictions Based on One-Day (Left) vs. $n$-Day (Right) Markov Matrix
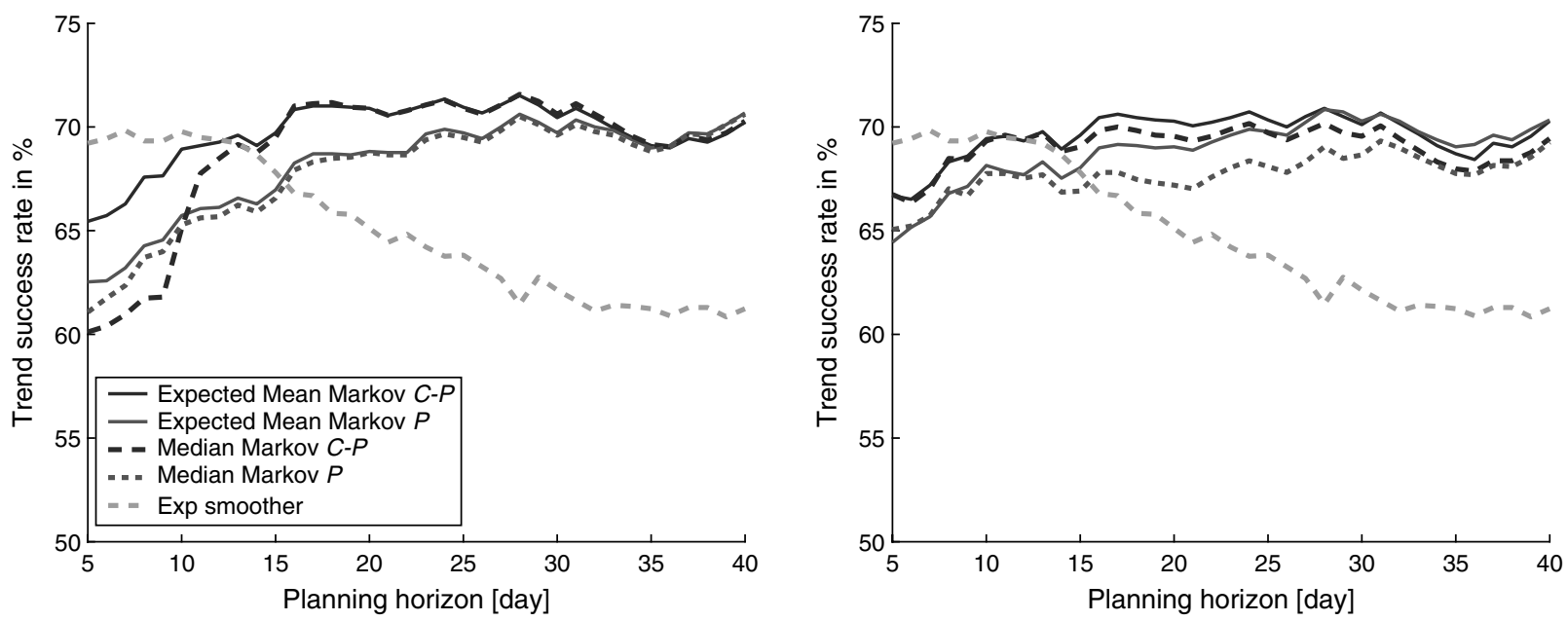
Figure 10 Daily Order Probability Estimation (Mean/Std) for the 10th, 25th, 50th, 75th, and 90th Percentile Using Different Predictors

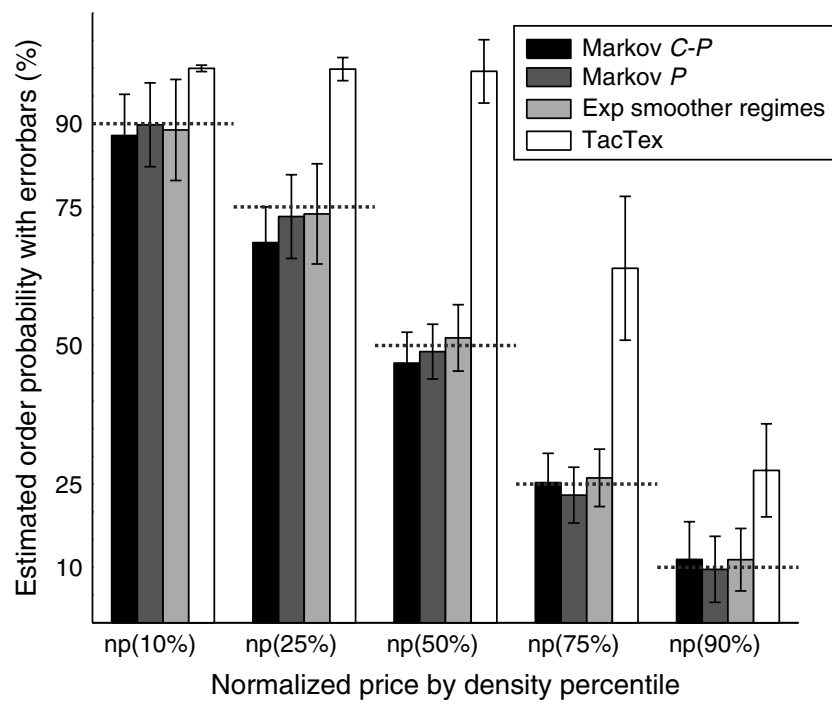

5.3.4. Estimation of Order Probability. Because we estimate the price trends from the accepted offers, an inverse relationship with order probability can be established. For example, on the normalized price curve a price representing a CDF of $10 \%$ corresponds to $90 \%$ order probability because there is $90 \%$ probability that a price at least as high as that price will be accepted. To test our assertion, we determined, using historical data, how many orders we would have won on each day if we had bid using estimated prices. ${ }^{8}$ For our experiments we estimated 2,200 (10 simulation runs each of 220 days) order probability curves for a sample market. Figure 10 shows the results of the experiments for the different predictors. The $y$-axis shows the estimated order probability, and the bars show the actual mean order probability and standard deviation. Our three predictors estimate the daily order probability well; the exponential smoother regime predictor tends to have larger standard deviations. The large errors in the TacTex probability estimates show the weakness of the linear approximation they use. It is the ability of regime models to estimate the whole price distribution instead of just the mean prices that produces these good estimates of order probabilities.

\subsection{Agent Performance}

The analysis presented so far demonstrates that our approach performs well with historical data. However, to make decisions in real time, the methods have to be dynamic and self-adjusting. We next evaluate the performance of our approach when used by an

${ }^{8}$ In TAC SCM, customers always accept the lowest bids. agent that plays against five other agents in real time in TAC SCM.

5.4.1. Experimental Setup. We implemented different prediction methods for short-term (tactical) and long-term (strategic) predictions and tested them in real time in our MinneTAC (Collins et al. 2009, 2010a) agent. The prediction methods we tested are linear predictor, exponential smoother, exponential smoother with regimes, Markov one-day predictor, and Markov $n$-day predictor.

The agents we used for our experiments have been obtained from the TAC SCM agent repository. ${ }^{9}$ We selected five of the finalists from the 2006 competition and an agent from the 2005 competition. The agents are (1) TacTex, from the University of Texas at Austin; (2) DeepMaize, from the University of Michigan; (3) PhantAgent, from the Politechnica University of Bucharest; (4) Maxon, from Xonar Inc.; (5) MinneTAC, from the University of Minnesota; and (6) RationalSCM, from the Australian National University.

Agent performance in TAC SCM is affected not only by the set of competing agents but also by random variations in supply, demand, and other market parameters. To compare different variations of our own agent without having to run a very large number of simulation runs, we used a version of the simulation server (Sodomka et al. 2007) that supports repeatable pseudo-random sequences of any individual market factor or combination of factors. The use of this server removes the profit variability because of agents facing different market conditions and enables us to test multiple variations of our MinneTAC agent under repeatable market conditions.

We ran $N_{G}=23$ simulations, each with a different pseudo-random sequence, using the base version of MinneTAC and then ran $N_{G}$ simulations with the same market factors, each using a different version of MinneTAC with different prediction models for tactical (order probability calculation when responding to RFQs) and strategic decisions (price and price trend prediction for sales quota and inventory holding decisions). At the strategic level we used different price prediction methods, namely an exponential smoother, an exponential smoother with regimes, a Markov prediction process with one-day, and a Markov prediction process with $n$-day predictions. At the tactical level we used two methods to calculate order probability, one based on a linear interpolation between the estimated minimum and maximum daily prices, the other an exponential smoother with economic regimes. 
Table 1 Experimental Results

\begin{tabular}{lccccc}
\hline & \multicolumn{5}{c}{ Mean profit/standard deviation (in million) } \\
\cline { 2 - 6 } $\begin{array}{l}\text { Experiment no. of } \\
\text { strategic: }\end{array}$ & 1 & 2 & 3 & 4 & 5 \\
\cline { 2 - 6 } Tactical: & ExpS & Markov- $P$ 1-day & ExpS with regimes & Markov- $P$ 1-day & Markov- $P$-day \\
\cline { 2 - 6 } Agent: & Linear & Linear & ExpS with regimes & ExpS with regimes & ExpS with regimes \\
\hline TacTex-06 & $8.752 / 5.682$ & $8.873 / 5.600$ & $9.302 / 5.343$ & $9.205 / 5.385$ & $9.061 / 5.331$ \\
DeepMaize-06F & $8.839 / 4.629$ & $8.713 / 4.846$ & $8.921 / 4.733$ & $8.318 / 4.181$ & $8.652 / 4.865$ \\
PhantAgent-06 & $8.049 / 5.422$ & $7.991 / 5.384$ & $8.029 / 5.425$ & $8.173 / 5.437$ & $7.953 / 5.247$ \\
Maxon-06F & $4.243 / 4.516$ & $3.767 / 4.288$ & $4.214 / 4.628$ & $4.019 / 4.181$ & $3.945 / 4.396$ \\
MinneTAC & $1.347 / 3.703$ & $1.813 / 4.017$ & $1.545 / 3.898$ & $2.117 / 3.764$ & $1.567 / 3.796$ \\
Rational-05 & $0.739 / 4.912$ & $0.669 / 4.692$ & $1.032 / 4.898$ & $1.305 / 4.527$ & $1.115 / 4.682$ \\
\hline
\end{tabular}

5.4.2. Real-Time Results. Our tests included five sets of 23 simulations each, one set for each different configuration of our MinneTAC agent using the same 23 pseudo-random sequences for each set.

As the primary measure of agent performance in Table 1 we show the mean total profit per agent. Table 1 shows that our MinneTAC agent always comes in fifth when competing against this set of agents. The performance of an agent depends upon its entire decision processes, which cover procurement, manufacturing, and sales. Our MinneTAC agent is somewhat weaker than its competitors in the procurement and manufacturing areas, but because our work is focused on sales performance, we are only interested in the relative performance of our MinneTAC agent under different sales strategies. The results of the experiments are as follows:

1. In the first experiment MinneTAC used a linear interpolation to determine the probability of order and an exponential smoother to predict price trends. The final mean profit is 1.347 million.

2. In the second experiment MinneTAC used again a linear interpolation to determine the probability of order and economic regimes (based on a repeated 1-day Markov prediction) to predict price trends. The final mean profit was 1.813 million.

3. The third experiment used an exponential smoother with regimes both to predict prices, and to determine the order probability, median prices, and price trends. It had a final mean profit of 1.545 million.

4. The fourth experiment used an exponential smoother with regimes for tactical decisions (determination of order probability) and a repeated oneday Markov predictor for strategic decisions (price and price trend prediction). The final mean profit for this experiment was 2.117 million, the best among the tested configurations.

5. The fifth experiment used an exponential smoother with regimes for tactical decisions and a Markov n-day prediction to determine price trends. Its final mean profit was 1.567 million.
We expected that the Markov $n$-day prediction would outperform the repeated one-day Markov prediction, as reported in \$5.3.2, but the outcome of our experiments shows the opposite. We attributed this to our off-line use of a separately trained Markov matrix for every day in the planning horizon; but because of the limited time available in real time we used only a 1, 10, and 20 day Markov prediction matrix.

Table 2 Summary of the Mathematical Notation Used in the Paper

\begin{tabular}{|c|c|}
\hline Symbol & Definition \\
\hline $\mathrm{np}$ & Normalized price \\
\hline$p(\mathrm{np})$ & Density of the normalized price \\
\hline$N$ & Number of Gaussians of Gaussian Mixture Model (GMM) \\
\hline$p\left(\mathrm{np} \mid \zeta_{i}\right)$ & Density of np given ith Gaussian of the GMM \\
\hline$P\left(\zeta_{i}\right)$ & Prior probability of $i$ th Gaussian of the GMM \\
\hline$P\left(\zeta_{i} \mid \mathrm{np}\right)$ & Posterior probability of ith Gaussian of GMM given $\mathrm{np}$ \\
\hline$\vec{\eta}(\mathrm{np})$ & $N$-dimensional vector of posterior probabilities of GMM \\
\hline M & Number of regimes \\
\hline$R_{k}$ & $k$ th regime, $k=1, \ldots, M$ \\
\hline$\hat{R}_{k}$ & Predicted $k$ th regime, $k=1, \ldots, M$ \\
\hline $\mathbf{P}(\zeta \mid r)$ & Conditional probability matrix ( $N$ rows and $M$ columns) \\
\hline$p\left(\mathrm{np} \mid R_{k}\right)$ & Density of normalized price np given regime $R_{k}$ \\
\hline$P\left(R_{k} \mid \mathrm{np}\right)$ & Probability of regime $R_{k}$ given normalized price $\mathrm{np}$ \\
\hline$\vec{P}\left(r_{d+n} \mid \widetilde{n p}_{d-1}\right)$ & $\begin{array}{l}\text { M-dimensional vector of probabilities of regimes on day } \\
d+n \text { given normalized price np on day } d-1\end{array}$ \\
\hline$\vec{P}\left(\hat{r}_{d+n} \mid \widetilde{n p}_{d-1}\right)$ & $\begin{array}{l}M \text {-dimensional vector of probabilities of predicted regimes } \\
\text { on day } d+n \text { given normalized price np on day } d-1\end{array}$ \\
\hline$P($ order $\mid \mathrm{np})$ & Probability of order given normalized price $\mathrm{np}$ \\
\hline$\widetilde{n p}^{\min }, \widetilde{n p}^{\max }$ & Smoothed minimum and maximum normalized price \\
\hline$\widetilde{\mathrm{np}}$ & Estimated mean normalized price \\
\hline$\widehat{\mathrm{np}}$ & Predicted mean normalized price \\
\hline$\alpha$ & Smoothing coefficient \\
\hline $\mathscr{C}$ & Set of all available component types \\
\hline $\mathscr{G}$ & Set of all goods (product types) \\
\hline$d$ & Current day \\
\hline$D_{d, g}$ & Total customer demand for good $g$ on day $d$ \\
\hline$D_{d, g}^{\mathrm{enf}}($ price $)$ & $\begin{array}{l}\text { Effective customer demand for good } g \text { on day } d \text { at } \\
\text { price price }\end{array}$ \\
\hline$\Phi$ & Total profit \\
\hline$A_{d, g}$ & Allocated sales quota for good $g$ on day $d$ \\
\hline$F$ & Daily production capacity of factory \\
\hline$O_{d, g}$ & Orders placed on day $d$ for goods $g$ \\
\hline$h$ & Planning horizon \\
\hline$\Pi$ & Stationary distribution of a Markov process \\
\hline
\end{tabular}


We performed regime and price density predictions for these three matrices, interpolating the missing prices between them. This assumes that the intermediate prices are linearly related to each other, which is not the case, because we actually expect prices to flatten out further into the future. We have performed an additional set of stylized experiments to explore the relative prediction quality of $n$-day Markov predictions versus one-day predictions (please see the online appendix for details). These show that the $n$-day approach is clearly superior for long-horizon predictions.

The results clearly show that mean profits increase when regimes are used for the purpose of pricing decisions. We conducted Wilcoxon signed rank test (Gibbons 1986) to assess the statistical significance because the data do not follow a normal distribution (the state of the simulations is wildly influenced by random number seeds resulting in many simulations producing no positive profits by any agent). Note that because the power of nonparametric tests is smaller than parametric tests, $p$-values smaller than 0.10 are considered adequate for statistical significance. The result of the tests show that there is no statistical difference in profits between experiment 3 and experiment 5 as compared to experiment 1 , but the profits are significantly higher in experiment $2(p=0.0523)$ and experiment $4(p=0.0061)$ as compared to experiment 1 . We further tested the difference in profits between experiment 4 and experiment 2 to see whether using regimes at the tactical level is beneficial as compared to using linear interpolation. The results indicated that the profits are significantly higher in experiment $4(p=0.0593)$ as compared to experiment 2 . The results show that the profit of our MinneTAC agent always has the lowest standard deviation, which indicates that our predictions are robust and stable.

The design of the simulation limits agents to about 12 seconds for each daily decision cycle, which must be allocated among procurement, manufacturing, and sales processes. The linear program described in $\$ 3.4 .1$ can require up to eight seconds but normally runs much faster. All of the regime models described in this paper run in a fraction of a second on modern desktop machines. Actual timings for a typical game in the 2010 competition are (mean, standard deviation) in seconds: exponential smoother regime prediction $(0.172,0.023)$; Markov regime prediction $(0.142,0.009)$; and LP solver $(0.186,0.083)$. The regime predictors are not only fast but very consistent, as evidenced by the low standard deviations.

\section{Conclusions and Future Work}

We proposed a versatile computational method based on both historical and observable data that can be used for tactical and strategic economic decision making by automated agents. The approach is based on fundamental economic principles, recognizing prevailing and predicted economic environments, or regimes, for making pricing and sales decisions. The computational process is completely data driven and no explicit classification of the market structure (monopoly versus competitive, etc.) is needed. A regime encapsulates a set of market parameters, with their appropriate range tailored to a specific market condition, thereby reducing the dimensionality of the parameter space. This results in a fast computational approach. Economic regimes provide comparatively more degrees of freedom than ordinary regression based approaches because the full price distribution is available for decision making. Availability of complete distributions and their trends allows a decision maker to choose an appropriate level of risk and supports estimation of other useful metrics such as order probabilities. Economic regimes are especially suited to make predictions in nonstationary environments where supply-demand relationship is highly dynamic. Economic regimes also provide an opportunity for niche learning; i.e., an agent is able to apply different approaches and actions when specific regimes are dominant.

We presented three different algorithms for dynamic identification of regimes and for prediction of regime distribution over a planning horizon. Our methods use knowledge of current and future regime distributions to facilitate tactical decision making, such as calculation of customer offer prices, and strategic decision making, such as allocation of resources over a planning horizon. Using the complete price distribution, instead of point estimates of prices, enables our approach to better account for price variance in decision making. Our choice of using only price and its associated quantity information to estimate regimes makes our approach applicable in real world competitive environments. In a real world market environment companies are able to observe competitors prices but have no access to internal data, such as costs, manufacturing capacity, and inventory positions.

If one has access to additional information in the real world, such as some simple measures of supply, demand, or both, then it would also make sense to incorporate these. An example could be to utilize the aggregated daily demand and compare it with the total available capacity of all manufacturers. A way to enter this into the model is to make the Markov transition matrices conditional on indicators of this demand capacity balance.

In future, we intend to apply our method in other domains where predicting price distributions may be fruitful, including B2B domains such as computer 
chips and components, $\mathrm{B} 2 \mathrm{C}$ domains such as Amazon .com and eBay.com, and in financial applications.

A real B2B domain we are currently working with is the Dutch Flower Auctions (DFA; Kambil and van Heck 1998). We have established a cooperation with the DFA and begun work to apply economic regimes to the flower market. The DFA play a vital role in maintaining the Netherlands's leadership in the flower industry; they serve as efficient centers for flower exchange between suppliers and buyers. In 2009, the DFA reported daily trades of more than 37.0 million cut flowers and 2.6 million potted plants, generating over 3.81 billion Euros in annual sales.

In the DFA, bidders decide which and how many flowers to bid on and at what price, and the auctioneers set initial prices, reserve prices, minimum lot sizes, and clock speeds. In the current practice, those auctioneering parameters are set up in a static manner. Realizing the opportunities offered by dynamic pricing in maximizing revenue (Zhao and Zheng 2000), and in order to increase the auction efficiency, we propose to move the auctioneer away from the current practice of static starting price setup to dynamic pricing using our method of economic regimes. Bidders could also use economic regimes to predict different market regimes and associated prices and align all elements of the supply chain accordingly, especially procurement and sales. An opportunistic buyer might bid low for certain flowers in an oversupply situation because he could sell them up to some threshold at a profit. On the other hand, if a scarcity situation is predicted, then the buyer might start bidding for certain flowers a few days earlier and store them in cold storage until the price reaches the highest point, e.g., Mother's day.

\section{Electronic Companion}

An electronic companion to this paper is available as part of the online version that can be found at http://isr.journal .informs.org/.

\section{References}

Bapna, R., P. Goes, A. Gupta, G. Karuga. 2008. Predicting bidders: Willingness to pay in online multi-unit ascending auctions: Analytical and empirical insights. INFORMS J. Comput. 20(3) 345-355.

Benisch, M., J. Andrews, N. Sadeh. 2006. Pricing for customers with probabilistic valuations as a continuous knapsack problem. Proc. 8th Internat. Conf. Electronic Commerce, Fredericton, NB, 38-46.

Benisch, M., A. Greenwald, I. Grypari, R. Lederman, V. Naroditskiy, M. Tschantz. 2004. Botticelli: A supply chain management agent designed to optimize under uncertainty. ACM Trans. Comp. Logic 4(3) 29-37.

Berry, P., K. Conley, M. Gervasio, B. Peintner, T. Uribe, N. Yorke-Smith. 2006. Deploying a personalized time management agent. Proc. Fifth Internat. Conf. Autonomous Agents and Multi-Agent Systems, Hakodate, Japan, 1546-1571.
Bichler, M., A. Gupta, W. Ketter. 2010. Designing smart markets. Inform. Systems Res. 21(4) 688-699.

Box, G., G. Jenkins. 1994. Time Series Analysis: Forecasting and Control, 3rd ed. Prentice Hall, Englewood Cliffs, NJ.

Brown, R. G., R. F. Meyer, D. A. D'Esopo. 1961. The fundamental theorem of exponential smoothing. Oper. Res. 9(5) 673-687.

Cachon, G., S. Netessine. 2004. Game theory in supply chain analysis. D. Simchi-Levi, S. D. Wu, and Z.-J. Shen, eds. Handbook of Quantitative Supply Chain Analysis Modeling in the eBusiness Era. Kluwer Academic Publishers, Norwell, MA, 13-66.

Chatzidimitriou, K. C., A. L. Symeonidis. 2009. Data-miningenhanced agents in dynamic supply-chain-management environments. IEEE Intelligent Systems 24(3) 54-63.

Chatzidimitriou, K. C., A. L. Symeonidis, I. Kontogounis, P. A. Mitkas. 2008. Agent Mertacor: A robust design for dealing with uncertainty and variation in SCM environments. Expert Systems with Appl. 35(3) 591-603.

Collins, J., W. Ketter, M. Gini. 2002. A multi-agent negotiation testbed for contracting tasks with temporal and precedence constraints. Internat. J. Electronic Commerce 7(1) 35-57.

Collins, J., W. Ketter, M. Gini. 2009. Flexible decision control in an autonomous trading agent. Electronic Commerce Res. Appl. 8(2) 91-105.

Collins, J., W. Ketter, M. Gini. 2010a. Flexible decision support in dynamic interorganizational networks. Eur. J. Inform. Systems 19(3) 436-448.

Collins, J., W. Ketter, N. Sadeh. 2010b. Pushing the limits of rational agents: The trading agent competition for supply chain management. AI Magazine 31(2) 63-80.

Collins, J., R. Arunachalam, N. Sadeh, J. Ericsson, N. Finne, S. Janson. 2005. The supply chain management game for the 2006 trading agent competition. Tech. Rep. CMU-ISRI-05-132, Carnegie Mellon University, Pittsburgh.

Dempster, A. P., N. M. Laird, D. B. Rubin. 1977. Maximum likelihood from incomplete data via the EM algorithm. J. Royal Stat. Soc. Series B 39(1) 1-38.

Elmaghraby, W., P. Keskinocak. 2003. Dynamic pricing in the presence of inventory considerations: Research overview, current practices, and future directions. Management Sci. 49(10) $1287-1309$.

Enders, W. 1995. Applied Econometric Time Series. Wiley, Hoboken, NJ.

Fan, M., J. Stallaert, A. B. Whinston. 2003. Decentralized mechanism design for supply chain organizations using an auction market. Inform. Systems Res. 14(1) 1-22.

Fine, S., Y. Singer, N. Tishby. 1998. The hierarchical hidden Markov model: Analysis and applications. Machine Learn. 32(1) 41-62.

Ghani, R. 2005. Price prediction and insurance for online auctions. Internat. Conf. Knowledge Discovery in Data Mining, Chicago, $411-418$.

Ghose, A., M. D. Smith, R. Telang. 2006. Internet exchanges for used books: An empirical analysis of product cannibalization and welfare impact. Inform. Systems Res. 17(1) 3-19.

Gibbons, J. D. 1986. Nonparametric statistical inference. Technometrics 28(3) 239-250.

Gray, J. A., D. E. Spencer. 1990. Price prediction errors and real activity: A reassessment. Econom. Inquiry 28(4) 658-681.

Gupta, A., D. O. Stahl, A. Whinston. 1997. The Internet: A future tragedy of the commons? H. Amman, B. Rustem, A. B. Whinston, eds. Computational Approaches to Economic Problems. Kluwer Academic Publishers, Dordrecht, The Netherlands, 347-361.

He, M., A. Rogers, X. Luo, N. R. Jennings. 2006. Designing a successful trading agent for supply chain management. Proc. Fifth Internat. Conf. Autonomous Agents and Multi-Agent Systems, Hakodate, Japan, 1159-1166.

Jordan, P. R., C. Kiekintveld, M. P. Wellman. 2007. Empirical gametheoretic analysis of the TAC supply chain game. Proc. Sixth Internat. Conf. Autonomous Agents and Multi-Agent Systems, Honolulu, 1188-1195. 
Kambil, A., E. van Heck. 1998. Reengineering the Dutch flower auctions: A framework for analyzing exchange organizations. Inform. Systems Res. 9(1) 1-19.

Kaplan, S., M. Sawhney. 2000. E-hubs: The new B2B marketplaces. Harvard Bus. Rev. 78(3) 97-103.

Karmarkar, N. 1984. A new polynomial-time algorithm for linear programming. Proc. Sixteenth Annual ACM Sympos. Theory Comput., ACM, New York, 302-311.

Ketter, W. 2007. Identification and prediction of economic regimes to guide decision making in multi-agent marketplaces. Ph.D. thesis, University of Minnesota, Twin-Cities.

Ketter, W., J. Collins, P. Reddy, C. Flath. 2011. The power trading agent competition. Tech. Rep. ERS-2011-011-LIS, RSM Erasmus University, Rotterdam, The Netherlands.

Ketter, W., J. Collins, M. Gini, A. Gupta, P. Schrater. 2006. Strategic sales management guided by economic regimes. E. van Heck et al., ed. Edited Volume of the 2nd Smart Business Network Initiative Discovery Event. Springer Verlag, Berlin, 113-125.

Ketter, W., J. Collins, M. Gini, A. Gupta, P. Schrater. 2009. Detecting and forecasting economic regimes in multi-agent automated exchanges. Decision Support Systems 47(4) 307-318.

Ketter, W., E. Kryzhnyaya, S. Damer, C. McMillen, A. Agovic, J. Collins, M. Gini. 2004. MinneTAC sales strategies for supply chain TAC. Internat. Conf. Autonomous Agents and Multi-Agent Systems, ACM, New York, 1372-1373.

Khreich, W., E. Granger, A. Miri, R. Sabourin. 2010. On the memory complexity of the forward-backward algorithm. Pattern Recognition Lett. 31(2) 91-99.

Kiekintveld, C., J. Miller, P. R. Jordan, L. F. Callender, M. P. Wellman. 2009. Forecasting market prices in a supply chain game. Electronic Commerce Res. Appl. 8(2) 63-77.

Kleindorfer, P. R., D. J. Wu. 2003. Integrating long-and short-term contracting via business-to-business exchanges for capitalintensive industries. Management Sci. 49(11) 1597-1615.

Kontogounis, I., K. C. Chatzidimitriou, A. L. Symeonidis, P. A. Mitkas. 2006. A robust agent design for dynamic SCM environments. Proc. 4th Hellenic Joint Conf. Artificial Intelligence (SETN), Heraklion, Greece, 127-136.

Kullback, S. 1959. Information Theory and Statistics. Dover Publications, New York.

Kullback, S., R. A. Leibler. 1951. On information and sufficiency. Ann. Math. Statist. 22(1) 79-86.

Lawrence, R. 2003. A machine learning approach to optimal bid pricing. 8th INFORMS Comput. Soc. Conf. Optim. Comput. Network Era, Phoenix, AZ, 1-22.

Mark, B., R. C. Perrault. 2006. Calo: Cognitive assistant that learns and organizes. Accessed on February 27, 2012, http:// www.ai.sri.com/ project/CALO.

Massey, C., G. Wu. 2005. Detecting regime shifts: The causes of under- and overestimation. Management Sci. 51(6) 932-947.

Muth, J. F. 1961. Rational expectations and the theory of price movements. Econometrica 29(3) 315-335.
Nagali, V., J. Hwang, D. S. M. Gaskins, M. Pridgen, T. Thurston, P. Mackenroth, D. Branvold, P. Scholler, G. Shoemaker. 2008. Procurement risk management (PRM) at Hewlett-Packard company. Interfaces 38(1) 51-60.

Nogales, F. J., J. Contreras, A. J. Conejo, R. Espinola. 2002. Forecasting next-day electricity prices by time series models. IEEE Trans. Power Systems 17(2) 342-348.

Osborn, D. R., M. Sensier. 2002. The prediction of business cycle phases: Financial variables and international linkages. National Inst. Econ. Rev. 182(1) 96-105.

Pardoe, D., P. Stone. 2004. Bidding for customer orders in TAC SCM: A learning approach. Workshop on Trading Agent Design and Analysis at AAMAS, New York, 52-58.

Pardoe, D., P. Stone. 2006. Tactex-05: A champion supply chain management agent. Proc. Twenty-First National Conf. Artificial Intelligence, AAAI, Boston, 1389-1394.

Pardoe, D., P. Stone. 2007. An autonomous agent for supply chain management. G. Adomavicius, A. Gupta, eds. Handbooks in Information Systems Series: Business Computing. Elsevier, Bingley, UK, 141-172.

Pauwels, K., D. Hanssens. 2002. Windows of change in mature markets. Eur. Marketing Acad. Conf., Braga, Portugal, 1-6.

Podobnik, V., A. Petric, G. Jezic. 2008. An agent-based solution for dynamic supply chain management. J. Universal Comput. Sci. 14(7) 1080-1104.

Sandholm, T. 2007. Expressive commerce and its application to sourcing: How we conducted $\$ 35$ billion of generalized combinatorial auctions. AI Magazine 28(3) 45-58.

Shannon, C. E. 1948. A mathematical theory of communication. Bell System Tech. J. 27(3) 379-423 and 623-656.

Shmueli, G. 2010. To explain or to predict? Statist. Sci. 25(3) 289-310.

Sodomka, E., J. Collins, M. Gini. 2007. Efficient statistical methods for evaluating trading agent performance. Proc. TwentySecond National Conf. Artificial Intelligence. Vancouver, Canada, 770-775.

Swaminathan, J. M., S. R. Tayur. 2003. Models for supply chains in e-business. Management Sci. 49(10) 1387-1406.

Swaminathan, J. M., S. F. Smith, N. M. Sadeh. 1998. Modeling supply chain dynamics: A multiagent approach. Decision Sci. 29(3) 607-632.

Titterington, D., A. Smith, U. Makov. 1985. Statistical Analysis of Finite Mixture Distributions. Wiley, New York.

Wang, S., W. Jank, G. Shmueli. 2008. Explaining and forecasting online auction prices and their dynamics using functional data analysis. J. Bus. Econom. Statist. 26(2) 144-160.

Zhang, X., W. K. Cheung. 2005. Learning global models based on distributed data abstractions. Nineteenth Internat. Joint Conf. Artificial Intelligence, Edinburgh, Scottland, 1645-1646.

Zhang, D., K. Zhao, C.-M. Liang, G. B. Huq, T.-H. Huang. 2004. Strategic trading agents via market modeling. SIGecom Exchanges 4(3) 46-55.

Zhao, W., Y.-S. Zheng. 2000. Optimal dynamic pricing for perishable assets with nonhomogeneous demand. Management Sci. 46(3) 375-388. 